Central Radio Propagation Laboratory
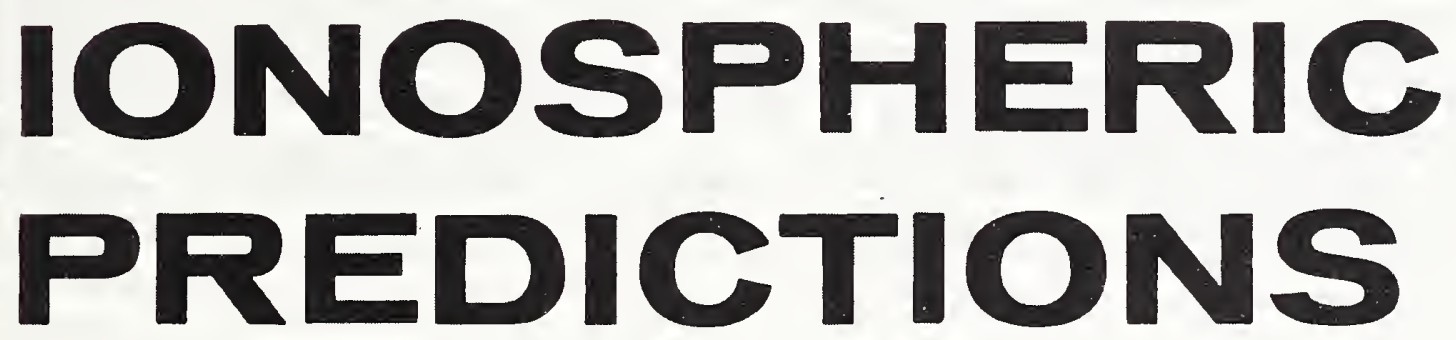

for

September

1963

TB 11-499-6/TO 31-3-28

U.S. DEPARTMENT of COMMERCE

National Bureau of Standards

Number 6/Issued June 1963 
Central Radio Propagation Laboratory

Ionospheric Predictions

\section{for September 1963}

\section{Number 6}

\section{Issued}

\section{June 1963}

[Formerly "Basic Radio Propagation Predictions," CRPL Series D.]

The CRPL Ionospheric Predictions are issued monthly as an aid in determining the best sky-wave frequencies over any transmission path, at any time of day, for average conditions for the month. Issued three months in advance, each issue provides tables of numerical coefficients that define the functions describing the predicted worldwide distribution of foF2 and $\mathrm{M}(3000) \mathrm{F} 2$ and maps for each even hour of universal time of MUF(Zero) F2 and MUF(4000) F2.

Note: Department of Defense personnel see back cover.

Use of funds for printing this publication approved by the Director of the Bureau of the Budget (June 19, 1961 ).

For sale by the Superintendent of Documents, U.S. Government Printing Office, Washington 25, D.C. Price 15 cents.

Annual subscription $(12$ issues) $\$ 1.50$ (50 cents additional for foreign mailing).

\section{National Bureau of Standards}

The functions of the National Bureau of Standards are set forth in the Act of Congress, March 3, 1901, as amended. These include the development and maintenance of the national standards of measurement and the provision of means and methods for making measurements consistent with these standards; the determination of physical constants and properties of materials; the development of methods and instruments fol testing materials, devices, and structures; advisory services to government agencies on scientific and tech- nical problems; invention and development of devices to serve special needs of the Government; and the development of standard practices, codes, and specifications. The work includes basic and applied research, development, engineering, instrumentation, testing, evaluation, calibration services, and various consultation and information services. The Bureau also serves as the Federal technical research center in a number of specialized fields.

\section{Central Radio Propagation Laboratory}

The Central Radio Propagation Laboratory at Boulder, Colorado, is the central agency of the Federal Government for the collection, analysis, and dissemination of information on propagation of radio waves at all ilequencies along the surface of the earth, in the atmosphere, and in space, and performs scientific studies looking toward new techniques for the efficient use and conservation of the radio spectrum. To carry out this responsibility, the CRPL-

1. Acts as the central agency for the conduct of basic research on the nature of radio waves, the pertinent properties of the media through which radio waves are transmitted, the interaction of radio waves with those media, and on the nature of radio noise and interference effects. This includes compilation of reports by other foreign and domestic agencies conducting research in this field and furnishing advice to government and nongovernment groups conducting propagation research.

2. Performs studies of specific radio propagation mechanisms and performs scientific studies looking toward the development of techniques for efficient use and conservation of the radiofrequency spectrum as part of its regular program or as requested by other government agencies. In an advisory capacity, coordinates studies in this area undertaken by other government agencies.

3. Furnishes advisory and consultative service on radio wave propagation, on radiofrequency utilization, and on radio systems problems to other organizations within the United States, public and private.

4. Prepares and issues predictions of radio wave propagation and noise conditions and warnings of disturbances in these conditions.

5. Acts as a central repository for data, reports, and information in the field of radio wave propagation.

6. Performs scientific liaison and exchanges data and information with other countries to advance knowledge of radio wave propagation and interference phenomena and spectrum conservation techniques, including that liaison required by international responsibilities and agreements. 


\section{Introduction}

The "Central Radio Propagation Laboratory Ionospheric Predictions" is the successor to the former "Basic Radio Propagation Predictions," CRPL Series D. To make effective use of these predictions, National Bureau of Standards Handbook 90, "Handbook for CRPL Ionospheric Predictions Based on Numerical Methods of Mapping," should be obtained from the Superintendent of Documents, U.S. Government Printing Ofrice, Washington 25, D.C., price 40 cents. This Handbook includes required additional data, nomographs and graphical aids, as well as methods for the use of the predictions. The Handbook supersedes the obsolete NBS Circular 465.

The basic prediction appears in tables 1 and 2, presenting predicted coefficients for foF 2 and $\mathrm{M}(3000) \mathrm{F} 2$ defining the numerical map functions describing the predicted worldwide variation of these characteristics. With additional auxiliary information, these coefficients may be used as input data for electronic computer programs solving specific high frequency propagation problems. The basic equations, their interpretation, and methods of using the numerical maps are described in two papers by W. B. Jones and R. M. Gallet, "The Representation of Diurnal and Geographic Variations of Ionospheric Data by Numerical Methods," Volume 66D, Number 4, JulyAugust 1962, pages 419-438, and "Methods for Applying Numerical Maps of Ionospheric Characteristics," Volume 66D, Number 6, November-December 1962, pages 649-662, both in the Journal of Research of the National Bureau of Standards, Section D. Radio Propagation. The predicted numerical map coeflicients of tables 1 and 2 may be purchased in the form of a tested set of punched cards. Write to the Prediction Service Section, Central Radio Propagation Laboratory, National Bureau of Standards, Boulder, Colorado, to arrange for the purchase of the set of punched cards and for further information and assistance in the application of computer methods and numerical prediction maps to specific propagation problems.

The graphical prediction maps, derived from the basic prediction, are provided for those unable to make use of an electronic computer. Figures 1 to 12 present world maps of MUF (Zero) F2 and MUF (4000) F2 for each even hour of universal time. Figures 13 to 16 present the same predictions for hours 00 and 12 universal time fol the North and South Polar areas. Predicted polar maps for each even hour of universal time may be obtained by special arrangements with the central Radio Propagation Laboratory. Handbook 90 describes methods for including regular E-F1 propagation. Figure $A$ is a graph of predicted and observed Zürich sunspot numbers which shows the recent trend of solar activity. Table A lists observed and predicted Zürich smoothed relative sunspot numbers and includes the sunpot number used for the current prediction.

Members of the U.S. Army, Navy, or Air Force desiring the Handbook and the Ionospheric Predictions should send requests to the proper service address; for the Navy: The Director, Naval Communications, Department of the Navy, Washington 25, D.C.; for the Air Force: Directorate of Command Control and Communications, Headquarters, United States Air Force, Washington 25, D.C., Attention: AFOCCAA. Army personnel should refer to the Handbook as TM 11-499 and to the monthly predictions as TB 11-499-( ), predictions for the month of September 1963 being distributed in June 1963 and designated TB 11-499-(6), and should requisition these through normal publication channels.

Information concerning the theory of radio wage propagation and such important problems as absorption, field intensity, lowest useful high frequencies, etc., is given in National Bureau of Standards Circular 462, "Ionospheric Radio Propagation." A revised work is in preparation which will be announced in the Ionospheric Prediction series when available. Additional information about radio noise may be found in C.C.I.R. Report Number 65, "Revision of Atmospheric Noise Data," International Telecommunication Union, Geneva, 1957.

Reports to this Laboratory of experience with these predictions would be appreciated. Correspondence should be addressed to the Predictions Services Section, Central Radio Propagation Laboratory, National Bureau of Standards, Boulder, Colorado. 
$\underline{\text { Table }} \underline{A}$

Observed and Predicted Zurich Smoothed Relative Sunspot Numbers

\begin{tabular}{|c|c|c|c|c|c|c|c|c|c|c|c|c|}
\hline Month & Jan. & Feb. & Mar. & Apr. & May & June & July & Aug. & Sept. & Oct. & Nov. & Dec. \\
\hline 1952 & $\begin{array}{c}43 \\
(53)\end{array}$ & $\begin{array}{c}42 \\
(51)\end{array}$ & $\begin{array}{c}39 \\
(52)\end{array}$ & $\begin{array}{c}36 \\
(52)\end{array}$ & $\begin{array}{c}34 \\
(52)\end{array}$ & $\begin{array}{c}32 \\
(52)\end{array}$ & $\begin{array}{c}31 \\
(51)\end{array}$ & $\begin{array}{c}29 \\
(49)\end{array}$ & $\begin{array}{c}28 \\
(46)\end{array}$ & $\begin{array}{c}28 \\
(43)\end{array}$ & $\begin{array}{c}27 \\
(38)\end{array}$ & $\begin{array}{c}26 \\
(33)\end{array}$ \\
\hline 1953 & $\begin{array}{c}24 \\
(30)\end{array}$ & $\begin{array}{c}22 \\
(29)\end{array}$ & $\begin{array}{c}20 \\
(27)\end{array}$ & $\begin{array}{c}19 \\
(24)\end{array}$ & $\begin{array}{c}17 \\
(22)\end{array}$ & $\begin{array}{c}15 \\
(21)\end{array}$ & $\begin{array}{c}13 \\
(20)\end{array}$ & $\begin{array}{c}12 \\
(18)\end{array}$ & $\begin{array}{c}11 \\
(18)\end{array}$ & $\begin{array}{c}10 \\
(17)\end{array}$ & $\begin{array}{c}9 \\
(16)\end{array}$ & $\begin{array}{c}7 \\
(15)\end{array}$ \\
\hline 1954 & $\begin{array}{c}6 \\
(14)\end{array}$ & $\begin{array}{c}6 \\
(12)\end{array}$ & $\begin{array}{c}4 \\
(11)\end{array}$ & $\begin{array}{c}3 \\
(10)\end{array}$ & $\begin{array}{c}4 \\
(10)\end{array}$ & $\begin{array}{c}4 \\
(9)\end{array}$ & $\begin{array}{c}5 \\
(8)\end{array}$ & $\begin{array}{c}7 \\
(8)\end{array}$ & $\begin{array}{c}8 \\
(8)\end{array}$ & $\begin{array}{c}8 \\
(10)\end{array}$ & $\begin{array}{c}10 \\
(10)\end{array}$ & $\begin{array}{c}12 \\
(11)\end{array}$ \\
\hline 1955 & $\begin{array}{c}14 \\
(12)\end{array}$ & $\begin{array}{l}16 \\
(14)\end{array}$ & $\begin{array}{c}20 \\
(14)\end{array}$ & $\begin{array}{c}23 \\
(13)\end{array}$ & $\begin{array}{c}29 \\
(16)\end{array}$ & $\begin{array}{c}35 \\
(18)\end{array}$ & $\begin{array}{c}40 \\
(22)\end{array}$ & $\begin{array}{c}46 \\
(27)\end{array}$ & $\begin{array}{c}55 \\
(30)\end{array}$ & $\begin{array}{c}64 \\
(31)\end{array}$ & $\begin{array}{c}73 \\
(35)\end{array}$ & $\begin{array}{c}81 \\
(42)\end{array}$ \\
\hline 1956 & $\begin{array}{c}89 \\
(48)\end{array}$ & $\begin{array}{c}98 \\
(53)\end{array}$ & $\begin{array}{l}109 \\
(60)\end{array}$ & $\begin{array}{l}119 \\
(68)\end{array}$ & $\begin{array}{l}127 \\
(77)\end{array}$ & $\begin{array}{l}137 \\
(89)\end{array}$ & $\begin{array}{l}146 \\
(95)\end{array}$ & $\begin{array}{c}150 \\
(105)\end{array}$ & $\begin{array}{c}151 \\
(119)\end{array}$ & $\begin{array}{c}156 \\
(135)\end{array}$ & $\begin{array}{c}160 \\
(147)\end{array}$ & $\begin{array}{c}164 \\
(150)\end{array}$ \\
\hline 1957 & $\begin{array}{c}170 \\
(150)\end{array}$ & $\begin{array}{c}172 \\
(150)\end{array}$ & $\begin{array}{c}174 \\
(150)\end{array}$ & $\begin{array}{c}181 \\
(150)\end{array}$ & $\begin{array}{c}186 \\
(150)\end{array}$ & $\begin{array}{c}188 \\
(150)\end{array}$ & $\begin{array}{c}191 \\
(150)\end{array}$ & $\begin{array}{c}194 \\
(150)\end{array}$ & $\begin{array}{c}197 \\
(150)\end{array}$ & $\begin{array}{c}200 \\
(150)\end{array}$ & $\begin{array}{c}201 \\
(150)\end{array}$ & $\begin{array}{c}200 \\
(150)\end{array}$ \\
\hline 1958 & $\begin{array}{c}199 \\
(150)\end{array}$ & $\begin{array}{c}201 \\
(150)\end{array}$ & $\begin{array}{c}201 \\
(150)\end{array}$ & $\begin{array}{c}197 \\
(150)\end{array}$ & $\begin{array}{c}191 \\
(150)\end{array}$ & $\begin{array}{c}187 \\
(150)\end{array}$ & $\begin{array}{c}185 \\
(150)\end{array}$ & $\begin{array}{c}185 \\
(150)\end{array}$ & $\begin{array}{c}184 \\
(150)\end{array}$ & $\begin{array}{c}182 \\
(150)\end{array}$ & $\begin{array}{c}181 \\
(150)\end{array}$ & $\begin{array}{c}180 \\
(150)\end{array}$ \\
\hline 1959 & $\begin{array}{c}179 \\
(150)\end{array}$ & $\begin{array}{c}177 \\
(150)\end{array}$ & $\begin{array}{c}174 \\
(150)\end{array}$ & $\begin{array}{c}169 \\
(150)\end{array}$ & $\begin{array}{c}165 \\
(146)\end{array}$ & $\begin{array}{c}161 \\
(143)\end{array}$ & $\begin{array}{c}156 \\
(141)\end{array}$ & $\begin{array}{c}151 \\
(142)\end{array}$ & $\begin{array}{c}146 \\
(141)\end{array}$ & $\begin{array}{c}141 \\
(139)\end{array}$ & $\begin{array}{c}137 \\
(137)\end{array}$ & $\begin{array}{c}132 \\
(137)\end{array}$ \\
\hline 1960 & $\begin{array}{c}129 \\
(136)\end{array}$ & $\begin{array}{c}125 \\
(135)\end{array}$ & $\begin{array}{c}122 \\
(133)\end{array}$ & $\begin{array}{c}120 \\
(130)\end{array}$ & $\begin{array}{c}117 \\
(125)\end{array}$ & $\begin{array}{c}114 \\
(120)\end{array}$ & $\begin{array}{c}109 \\
(118)\end{array}$ & $\begin{array}{c}102 \\
(115)\end{array}$ & $\begin{array}{c}98 \\
(110)\end{array}$ & $\begin{array}{c}93 \\
(108)\end{array}$ & $\begin{array}{c}88 \\
(105)\end{array}$ & $\begin{array}{c}84 \\
(100)\end{array}$ \\
\hline 1961 & $\begin{array}{c}80 \\
(100)\end{array}$ & $\begin{array}{c}75 \\
(90)\end{array}$ & $\begin{array}{c}69 \\
(90)\end{array}$ & $\begin{array}{c}64 \\
(90)\end{array}$ & $\begin{array}{c}60 \\
(85)\end{array}$ & $\begin{array}{c}56 \\
(85)\end{array}$ & $\begin{array}{c}53 \\
(80)\end{array}$ & $\begin{array}{c}52 \\
(75)\end{array}$ & $\begin{array}{c}52 \\
(70)\end{array}$ & $\begin{array}{c}51 \\
(70)\end{array}$ & $\begin{array}{c}50 \\
(65)\end{array}$ & $\begin{array}{c}49 \\
(60)\end{array}$ \\
\hline 1962 & $\begin{array}{c}45 \\
(60)\end{array}$ & $\begin{array}{c}42 \\
(50)\end{array}$ & $\begin{array}{c}40 \\
(48)\end{array}$ & $\begin{array}{c}39 \\
(45)\end{array}$ & $\begin{array}{c}39 \\
(42)\end{array}$ & $\begin{array}{c}38 \\
(37)\end{array}$ & $\begin{array}{c}36 \\
(34)\end{array}$ & $\begin{array}{c}34 \\
(31)\end{array}$ & $\begin{array}{c}31 \\
(29)\end{array}$ & $(28)$ & $(27)$ & (34) \\
\hline 1963 & (31) & (28) & (26) & (25) & (25) & (25) & (23) & (21) & $(20) *$ & & & \\
\hline 1964 & & & & & & & & & & & & \\
\hline
\end{tabular}

Note: Final numbers are listed through June 1963, the succeeding values being based on provisional data. The predicted numbers are in parentheses.

$*$ Number used for predictions in this issue. 


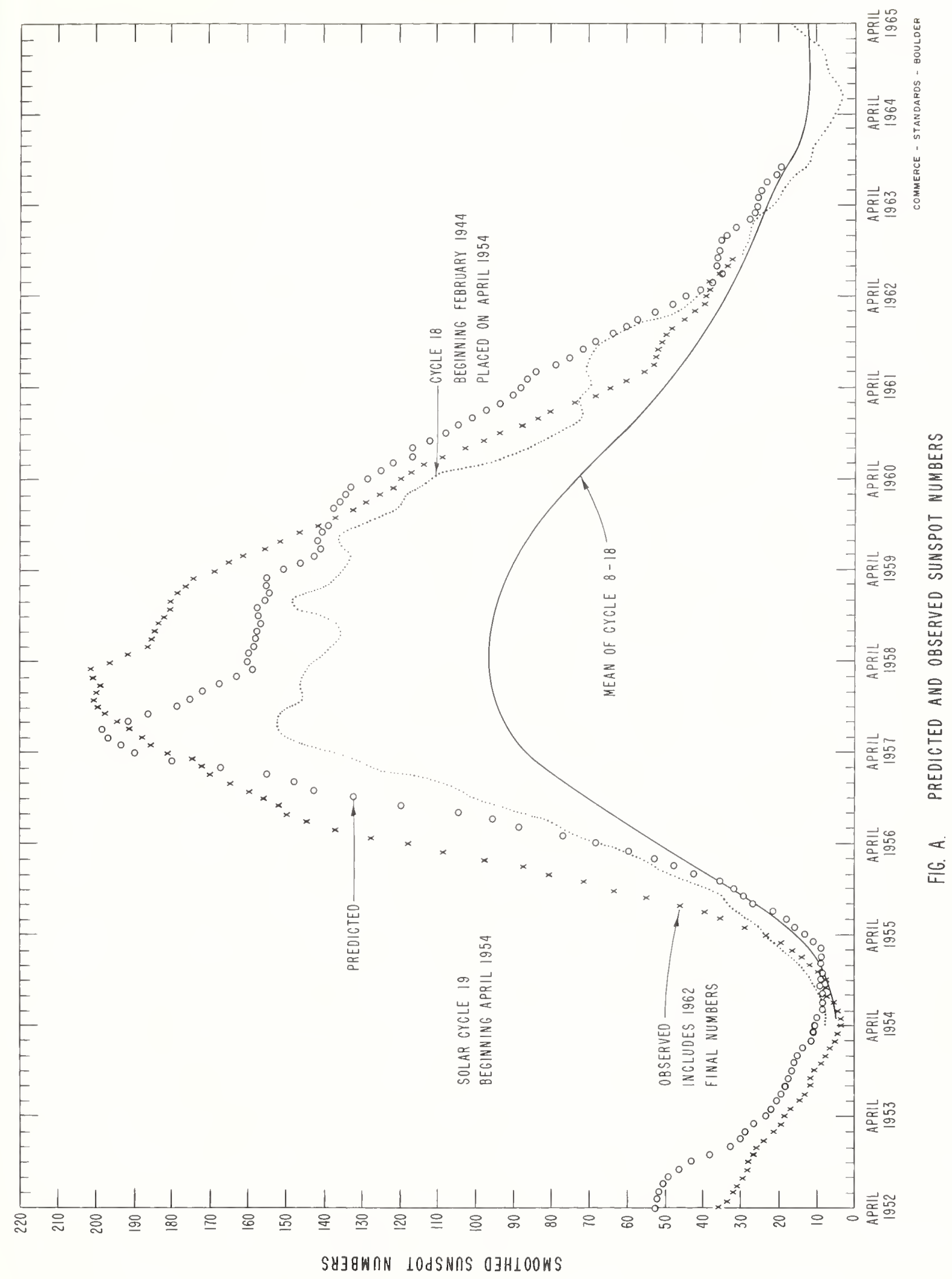




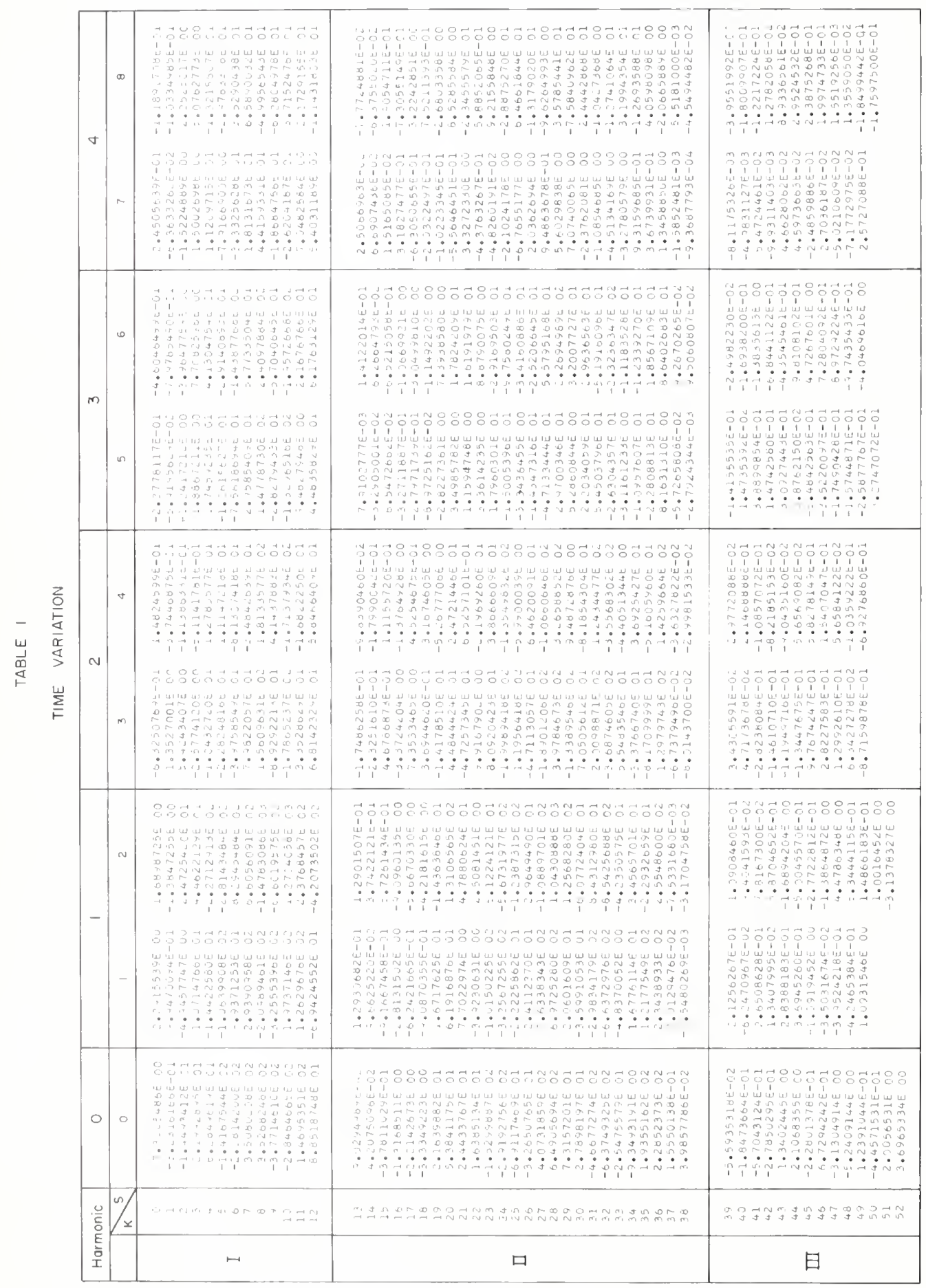

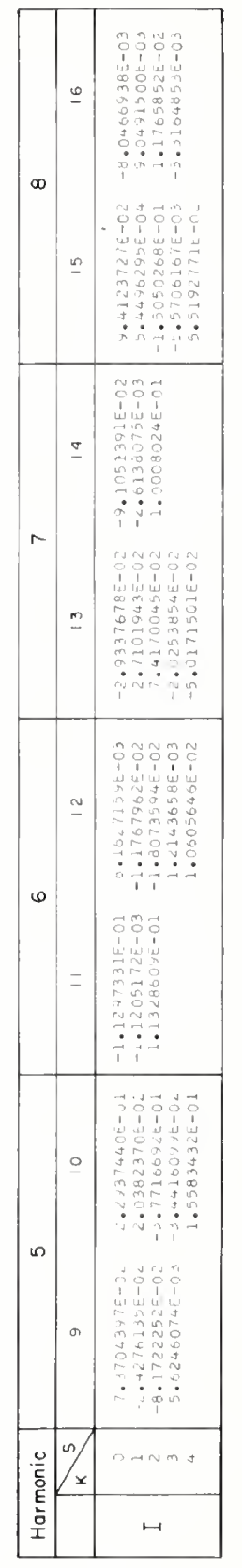

NOILVIYYA

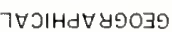

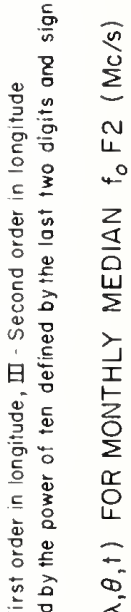

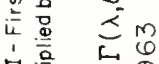

品器告

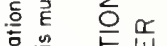

票之盀

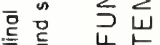

崖品

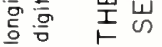

듬 志

亭京玄

言㟔

然

离高

衣吉

흘

몬

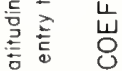

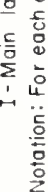

崩 


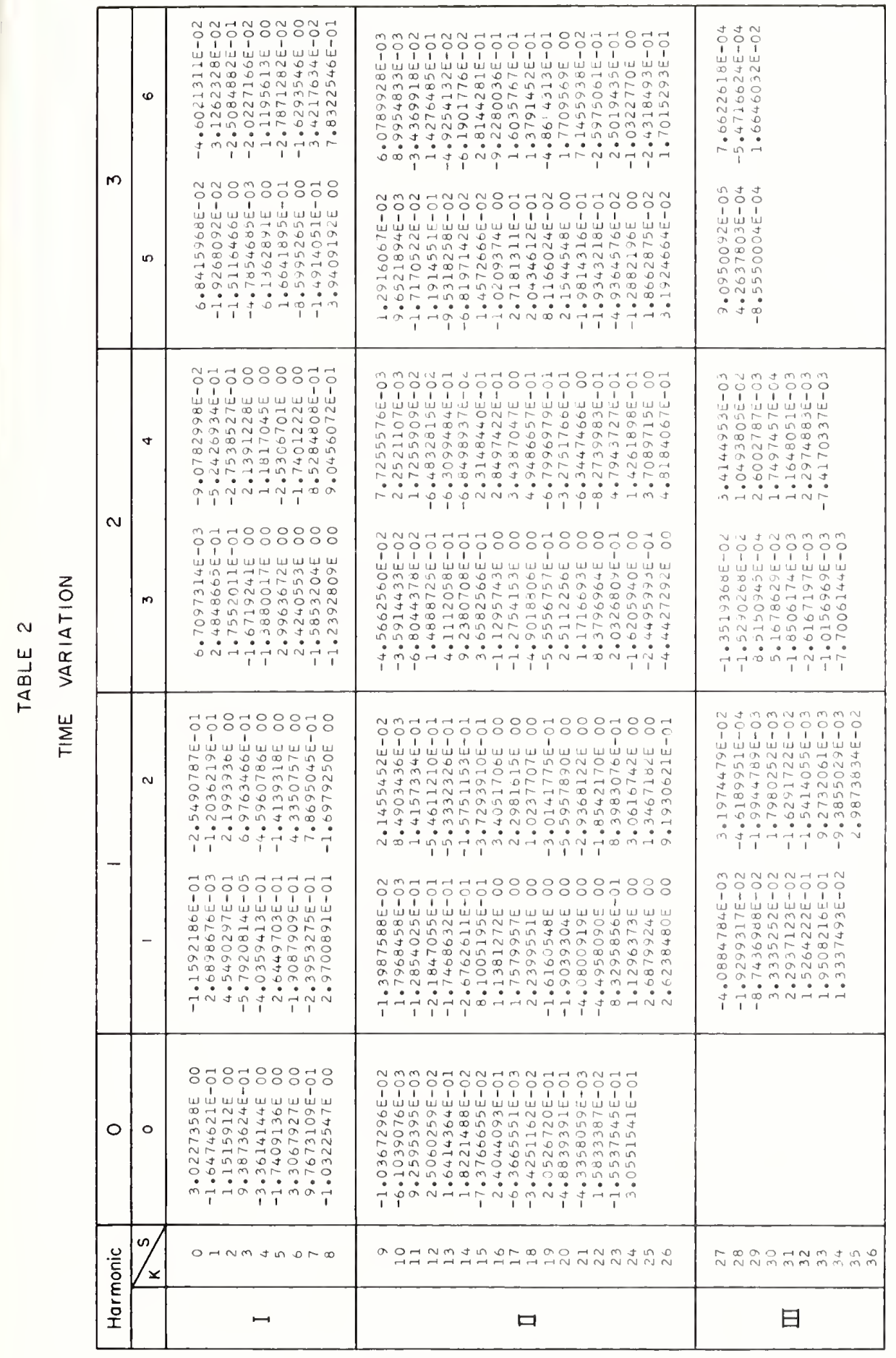

NOIL

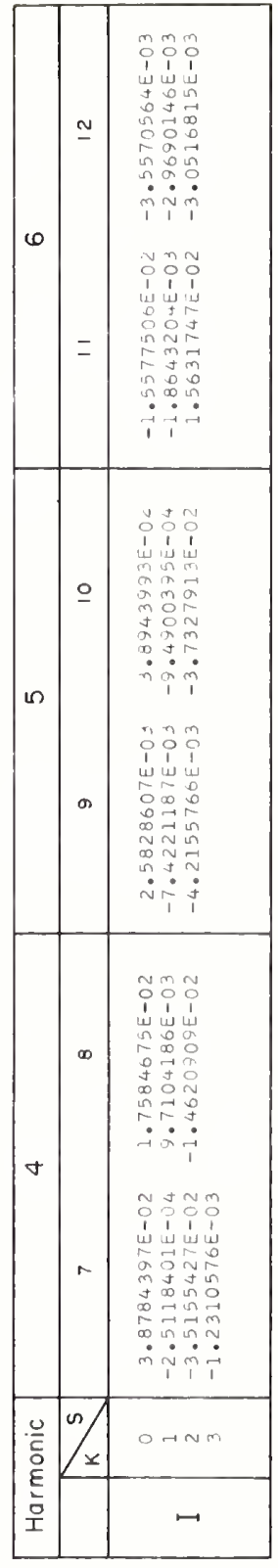

NOI $\perp \forall \mid \forall \forall \wedge$

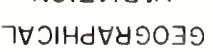

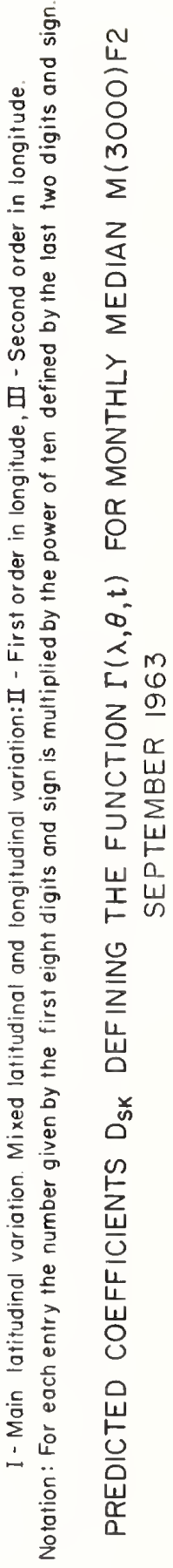



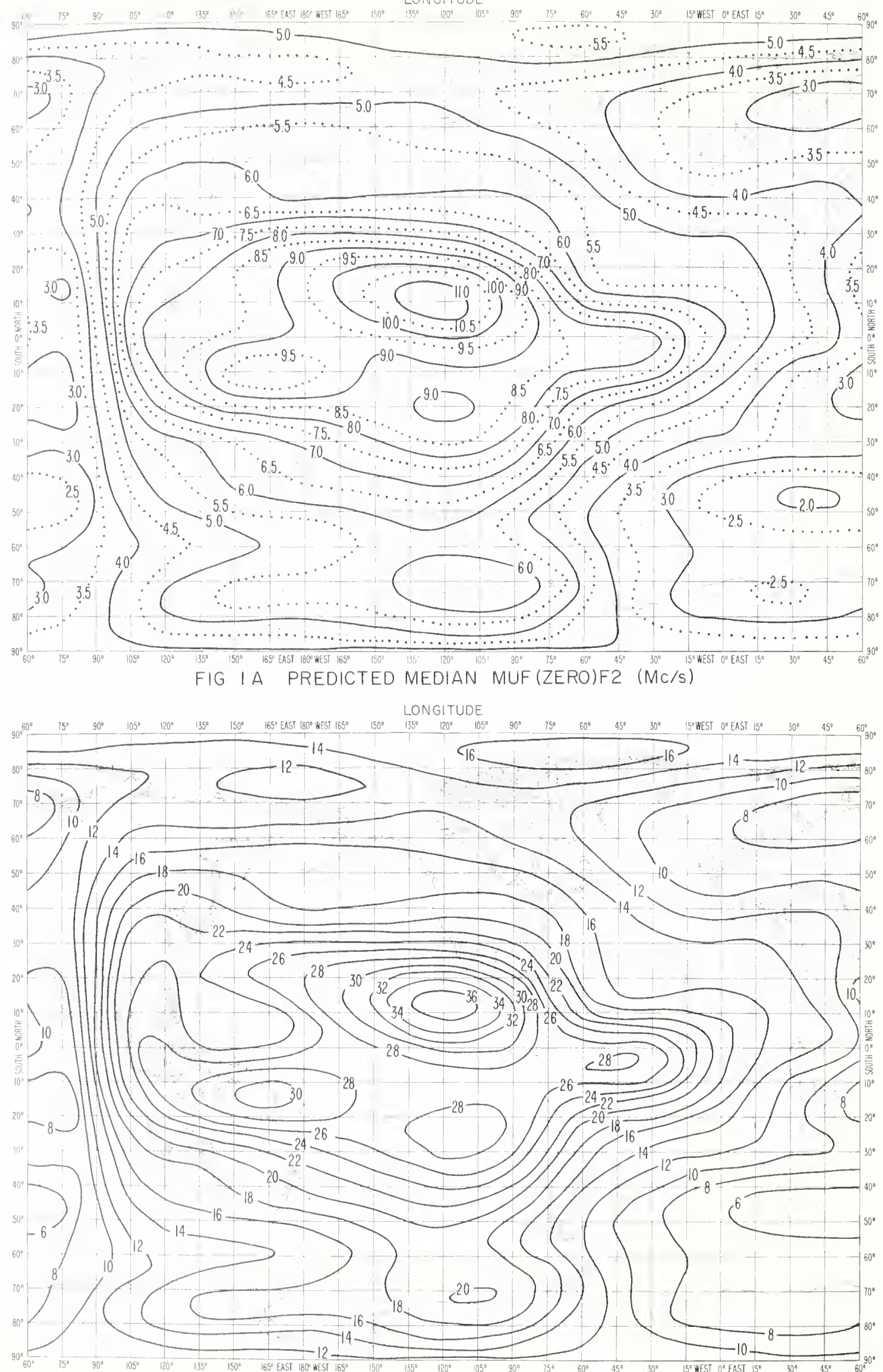


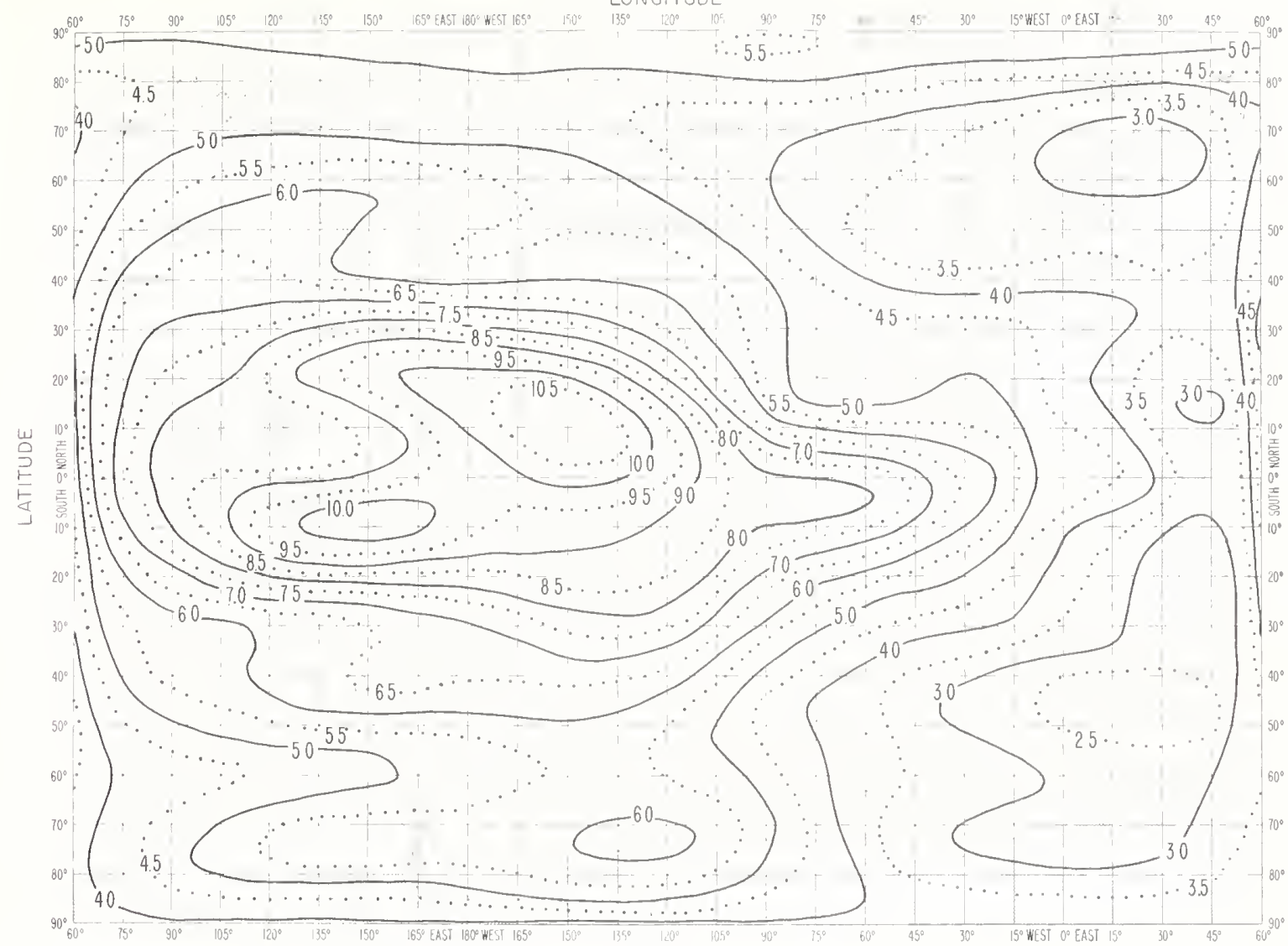

FIG 2A. PREDICTED MEDIAN MUF(ZERO)F2 ( $\mathrm{Mc} / \mathrm{s})$

LONGITUOE

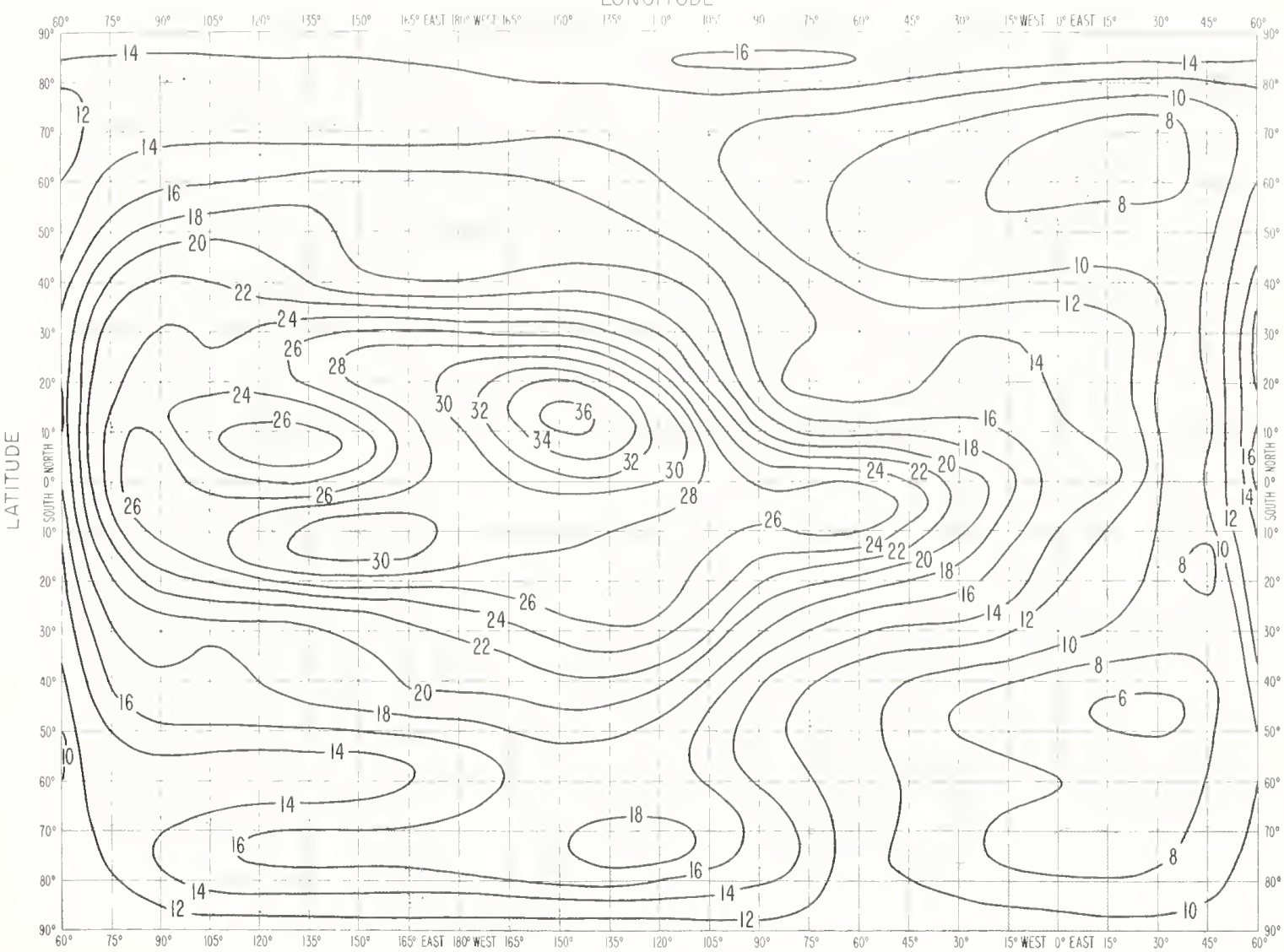

FIG $2 \mathrm{~B}$ PREDICTED MEDIAN MUF (4000)F2 ( $\mathrm{Mc} / \mathrm{s})$ 


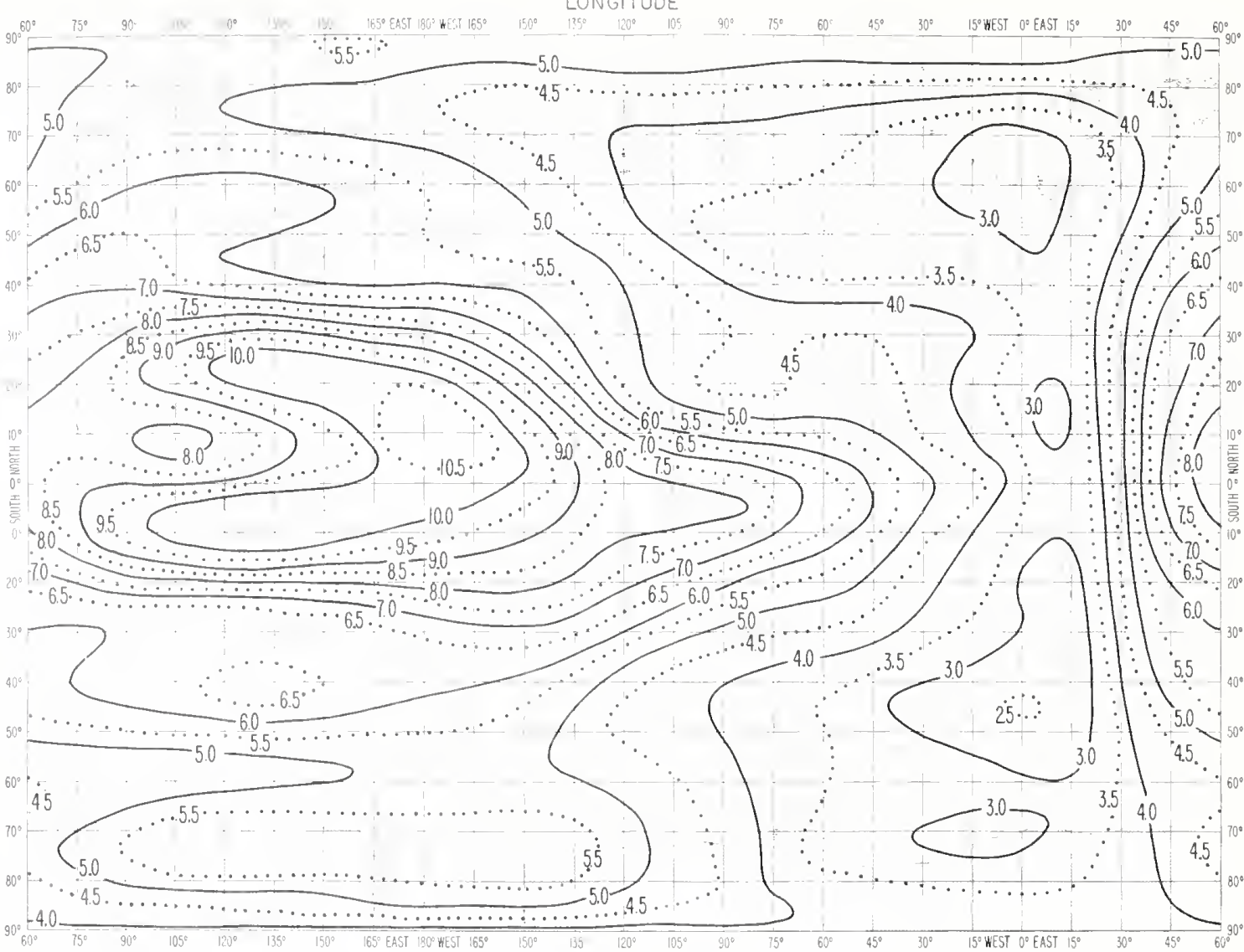

FIG 3 A. PREDICTED MEDIAN MUF(ZERO)F2 (Mc/s)

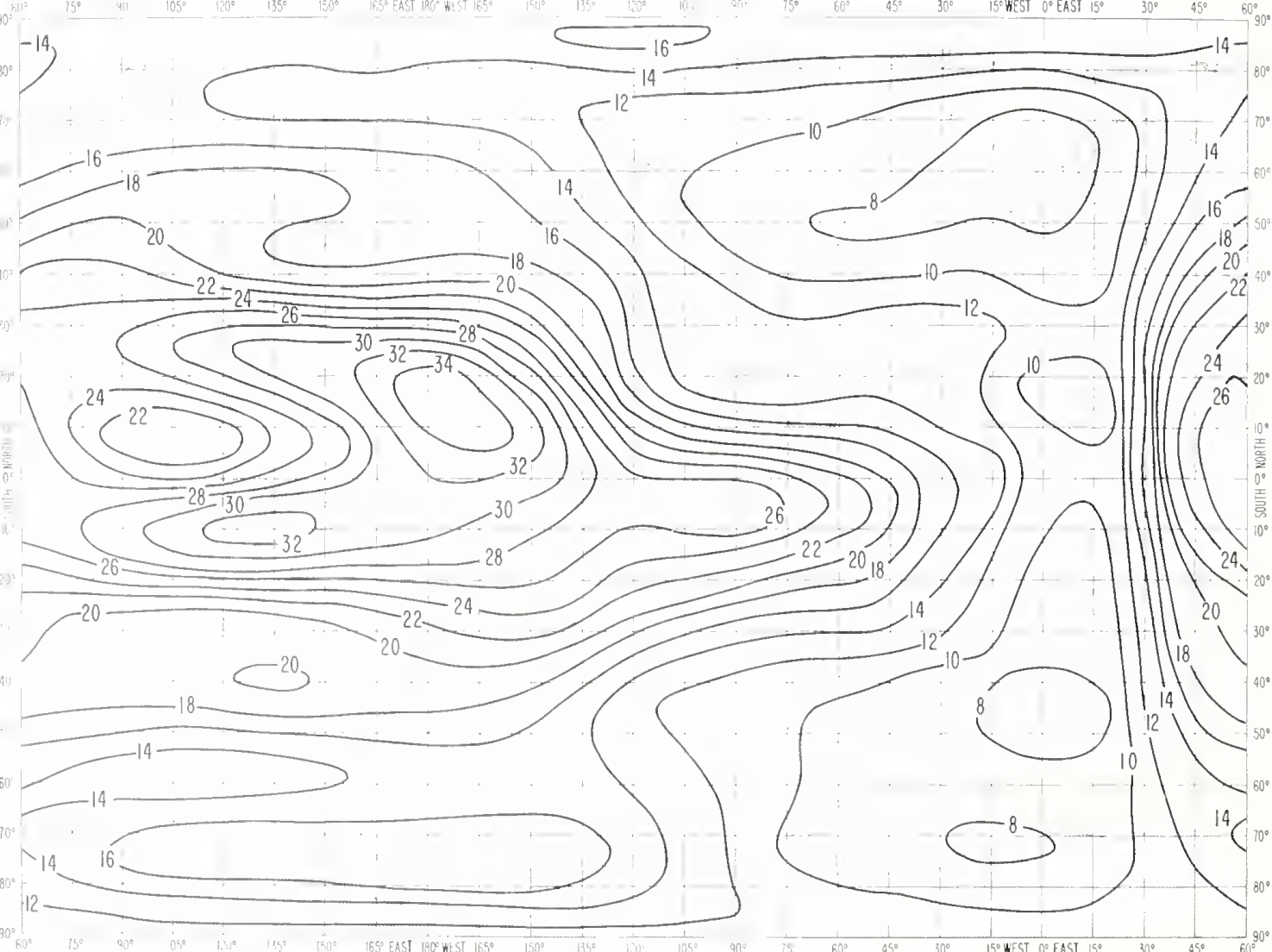




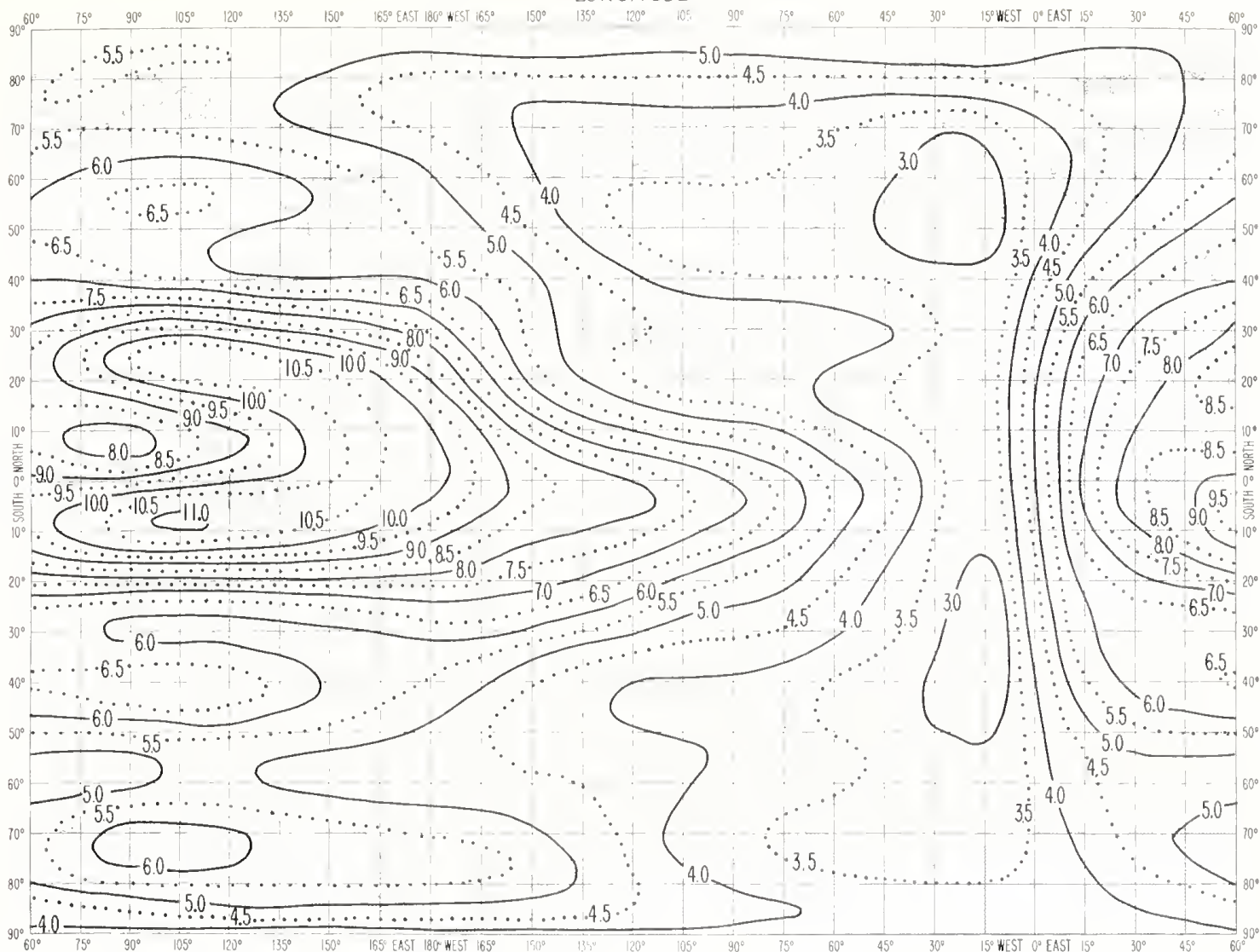

FIG 4 A PREDICTED MEDIAN MUF (ZERO)F2 ( $\mathrm{Mc} / \mathrm{s})$

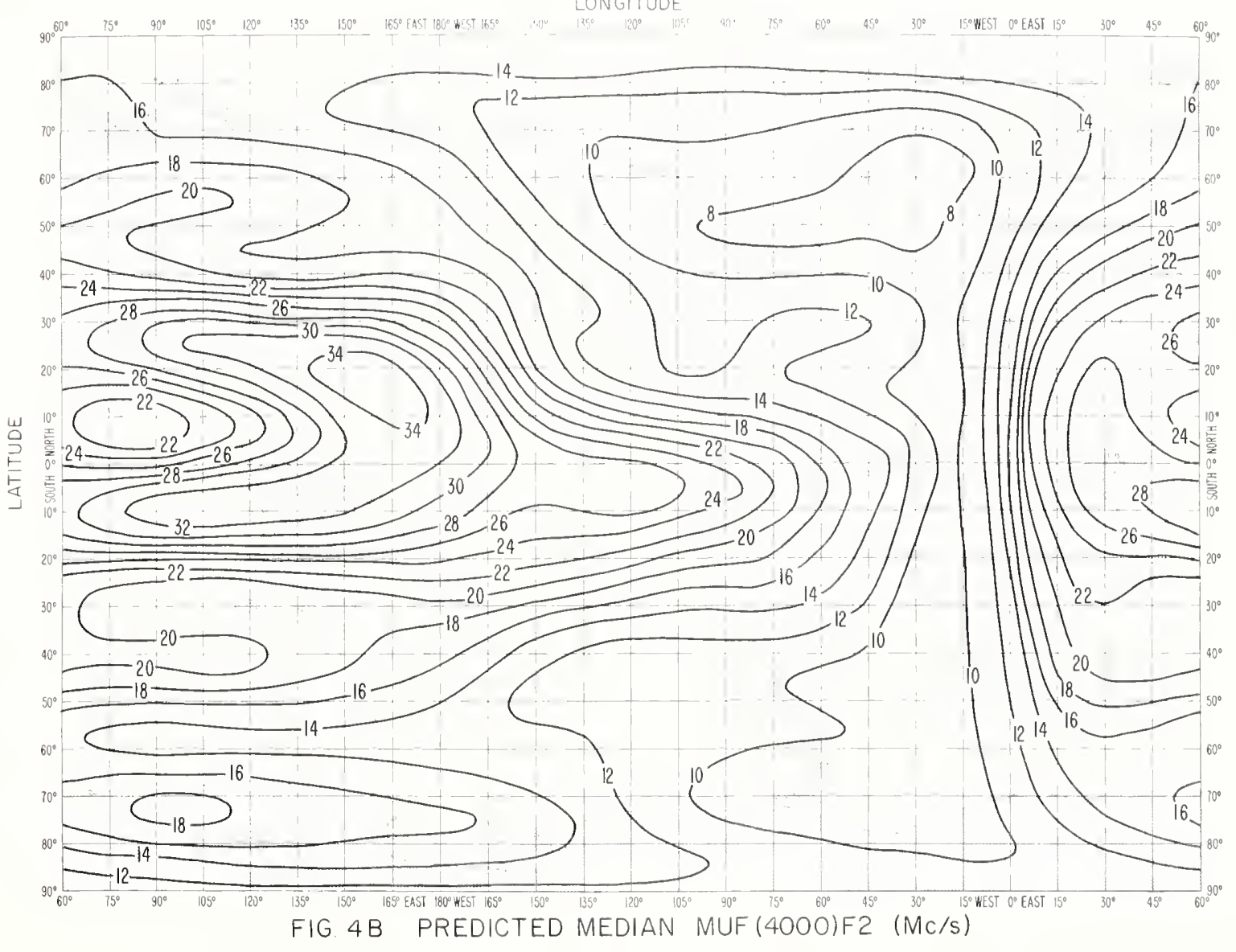




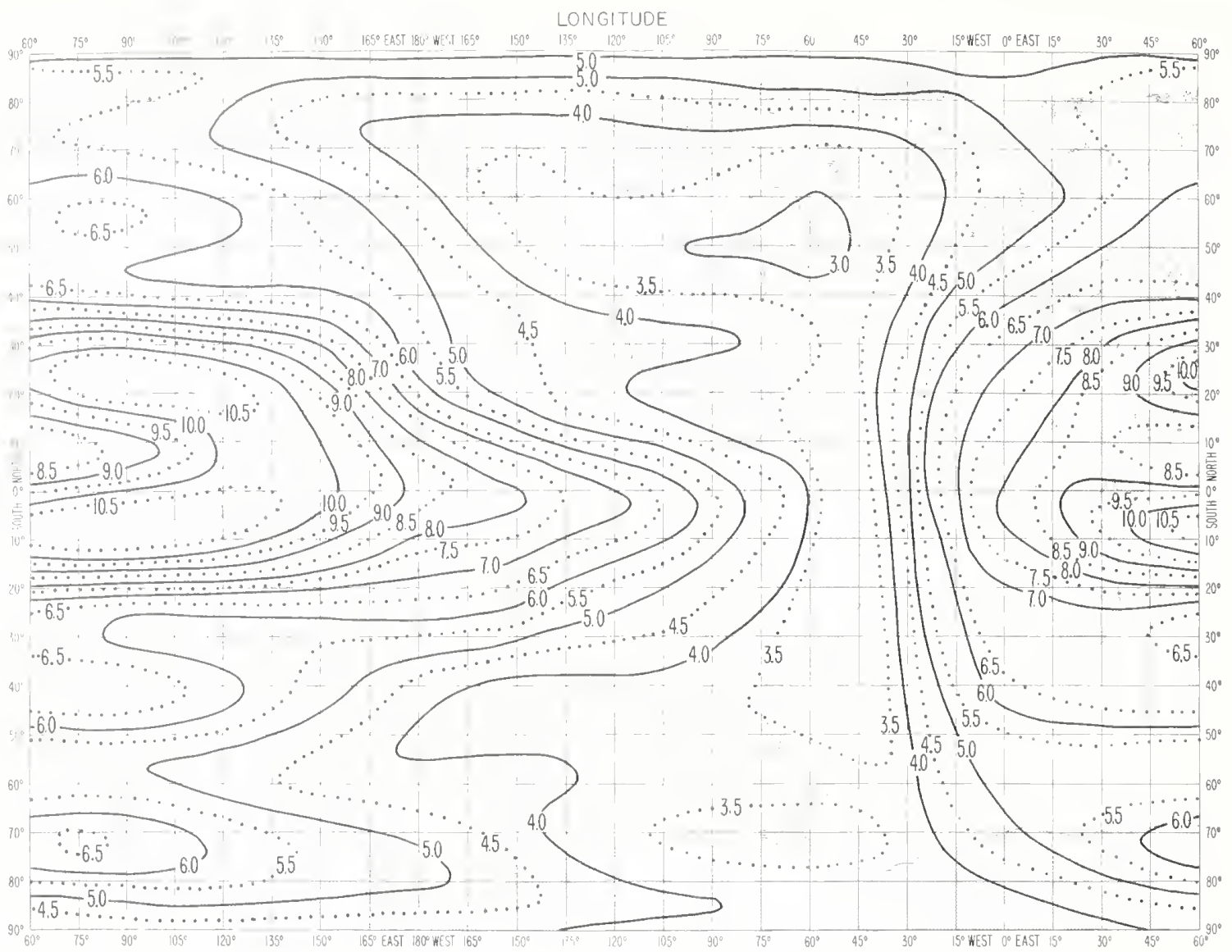

FIG 5 A PREDICTED MEDIAN MUF(ZERO)F2 ( $\mathrm{Mc} / \mathrm{s}$ )

LONGITUDE

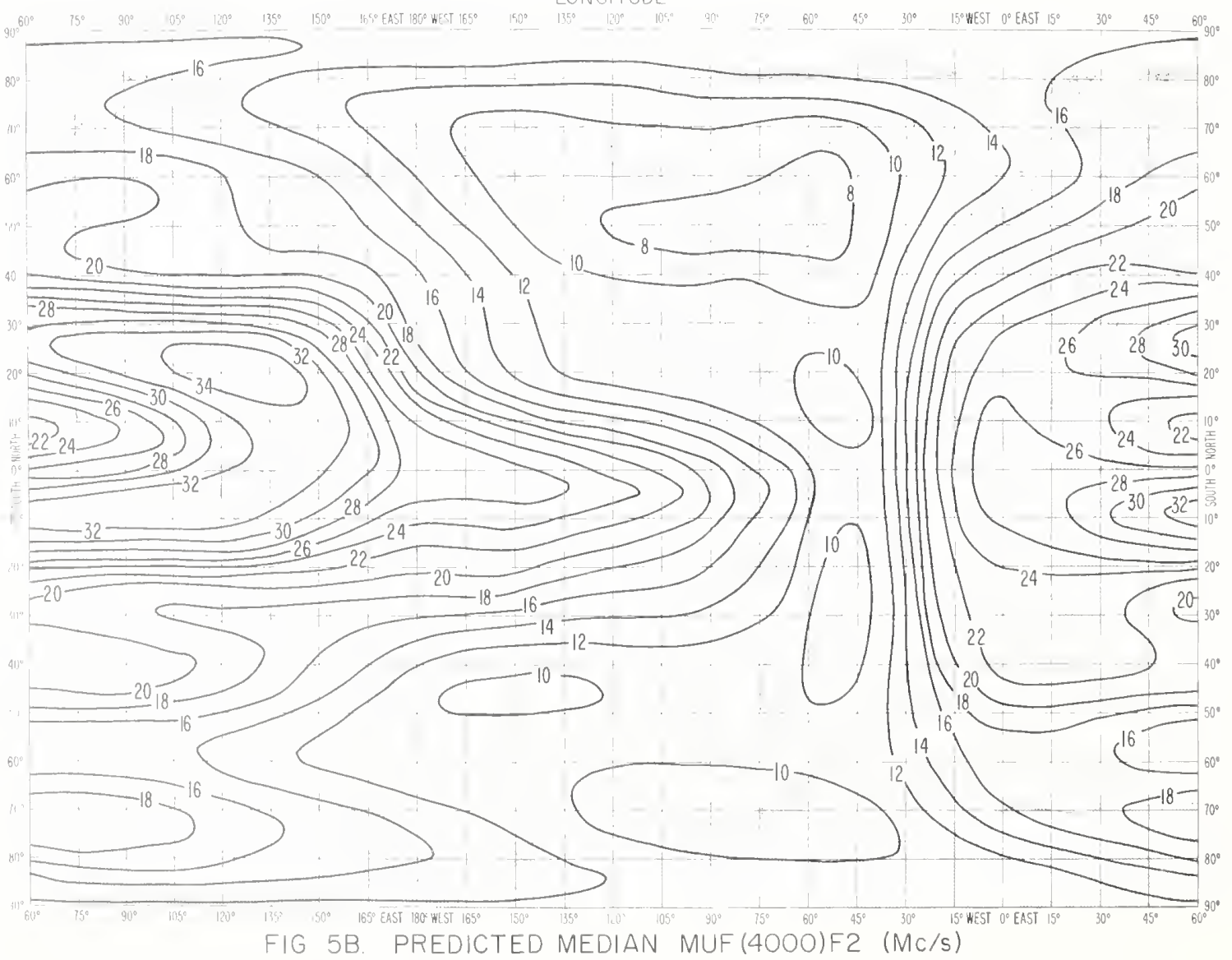




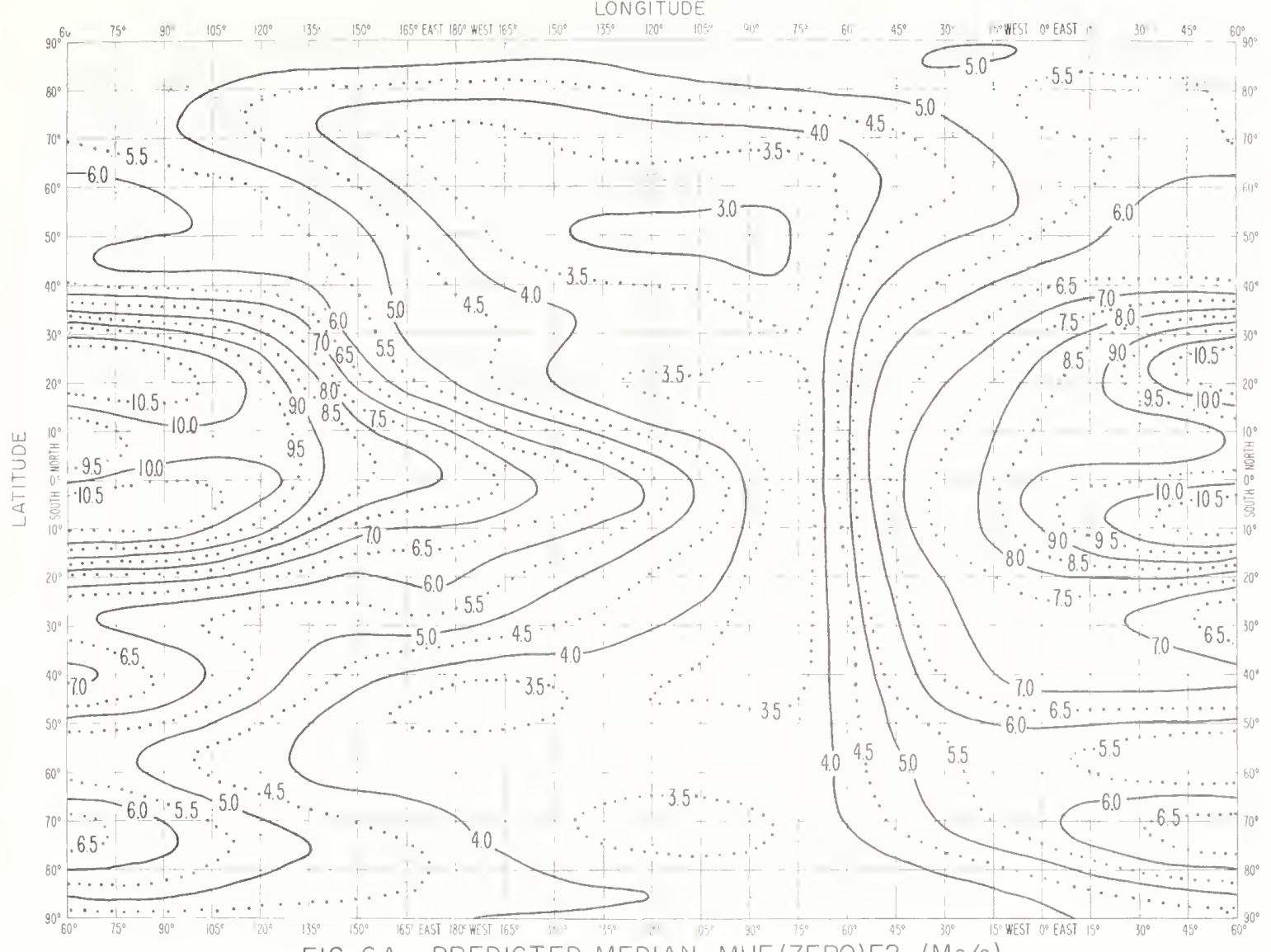

FIG. 6A. PREDICTED MEDIAN MUF (ZERO)F2 ( $\mathrm{Mc} / \mathrm{s})$

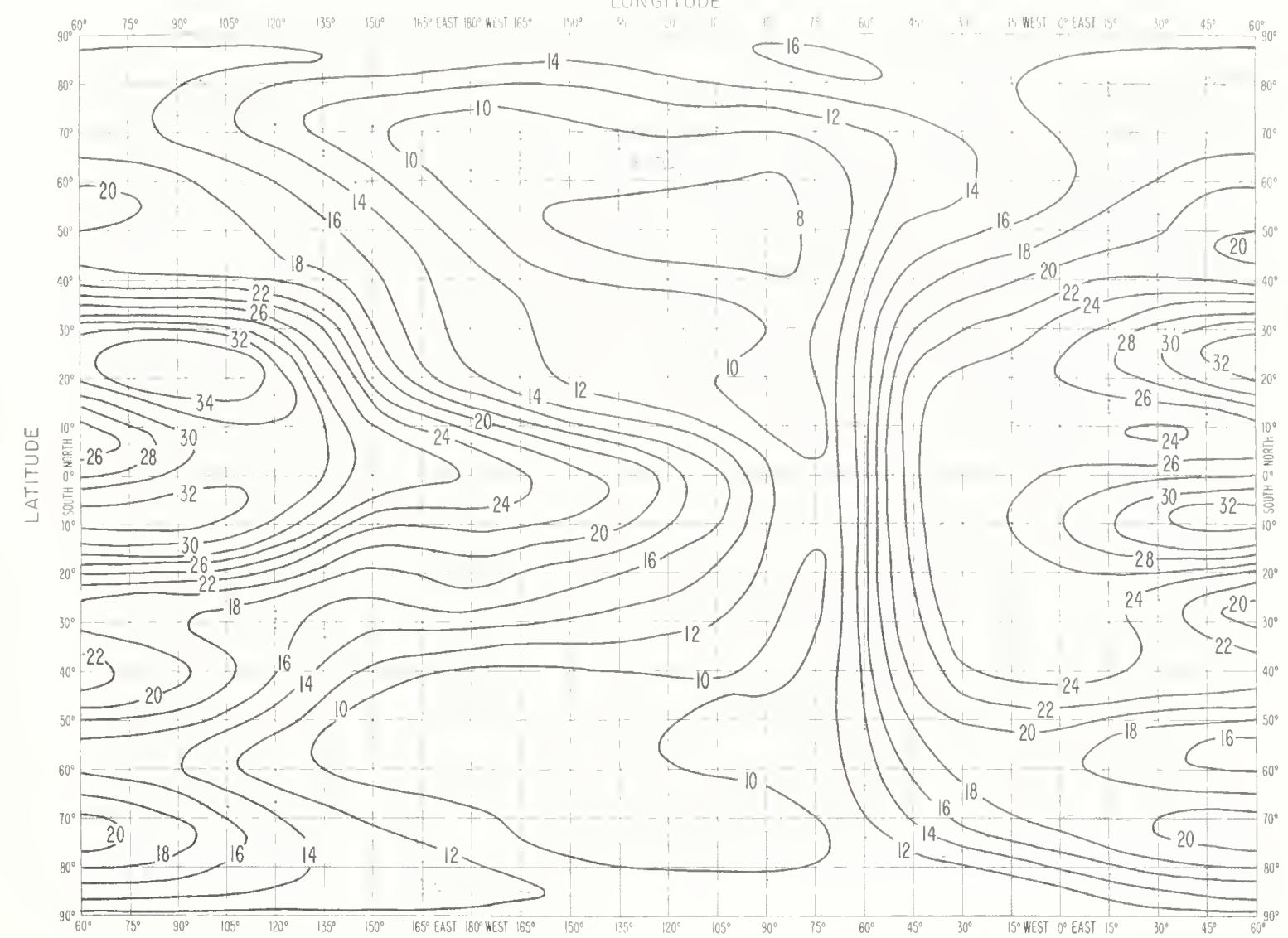

FIG $6 \mathrm{~B}$ PREDICTED MEDIAN MUF (4000)F2 ( $\mathrm{Mc} / \mathrm{s})$ 


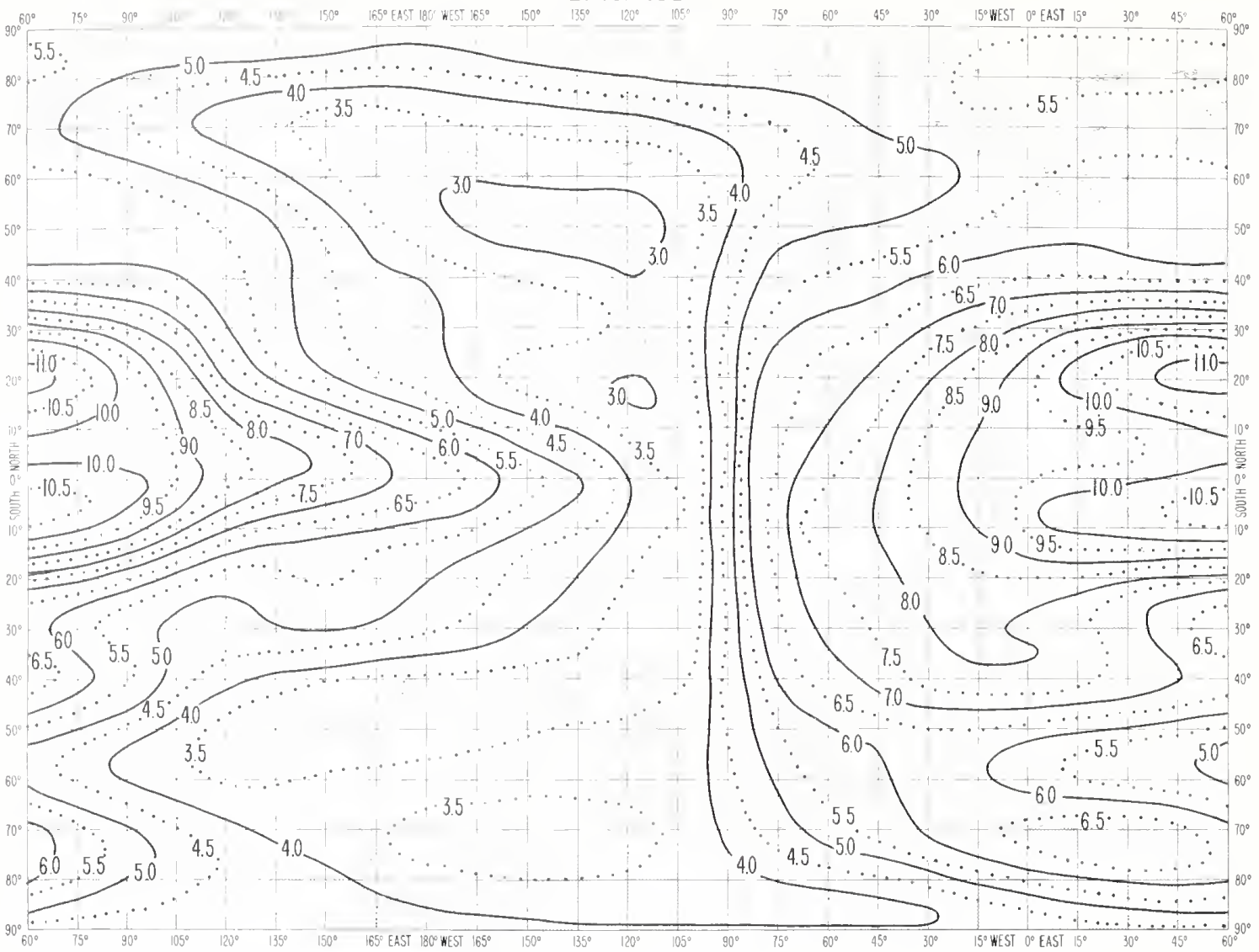
FIG. TA. PREDICTED MEDIAN MUF (ZERO)F2 ( $\mathrm{Mc} / \mathrm{s})$

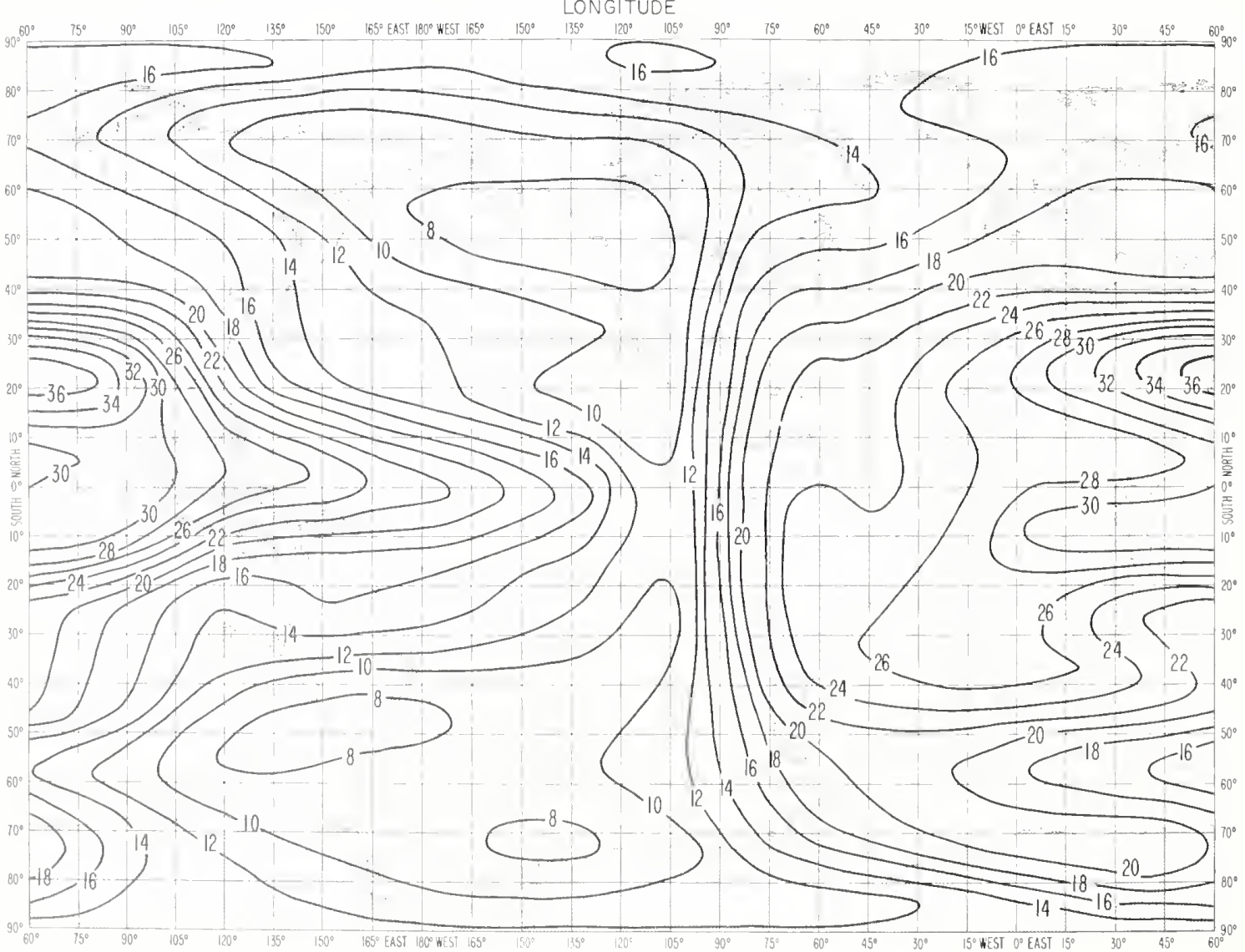




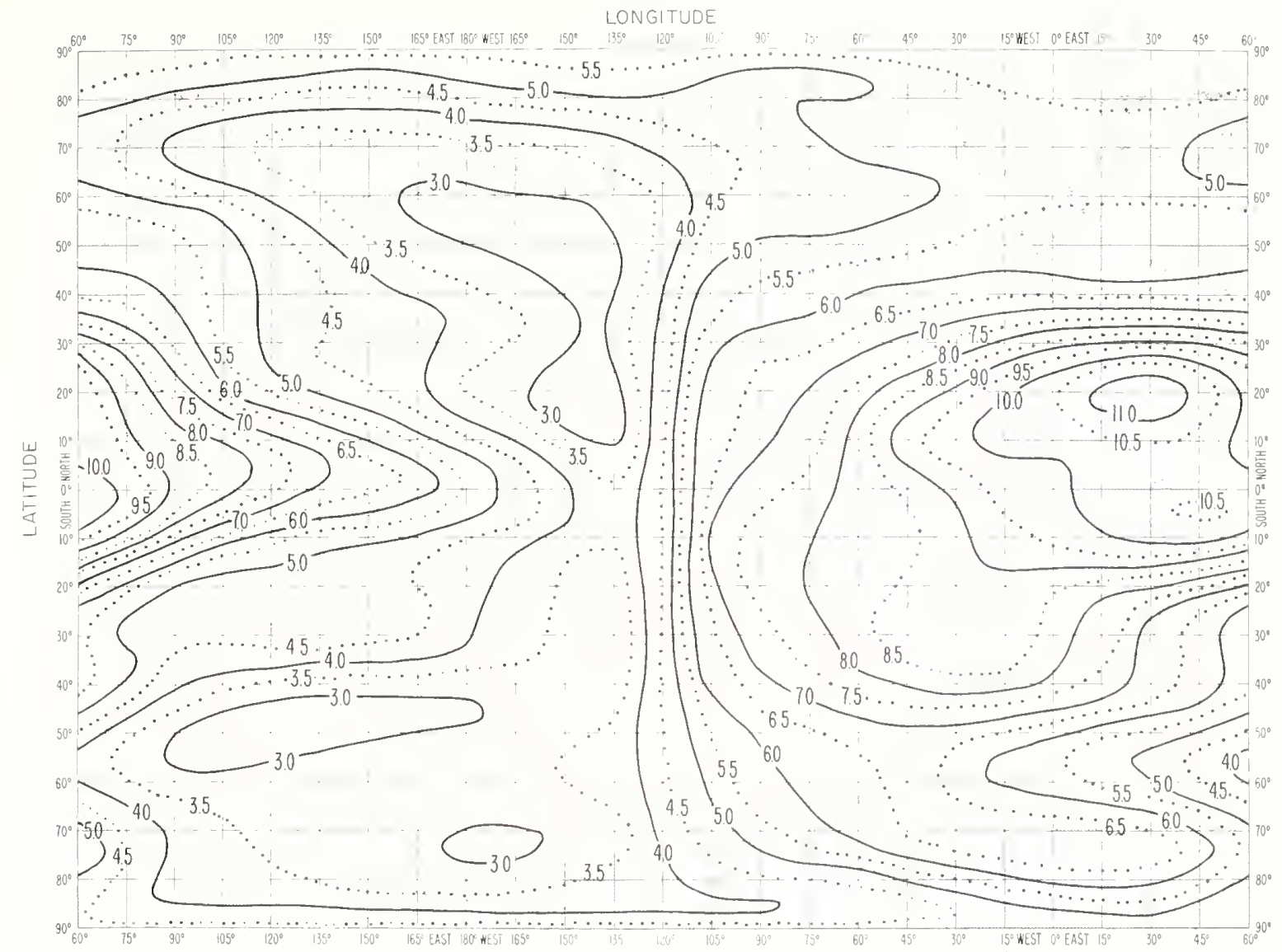

FIG 8A. PREDICTED MEDIAN MUF (ZERO)F2 ( $\mathrm{Mc} / \mathrm{s})$

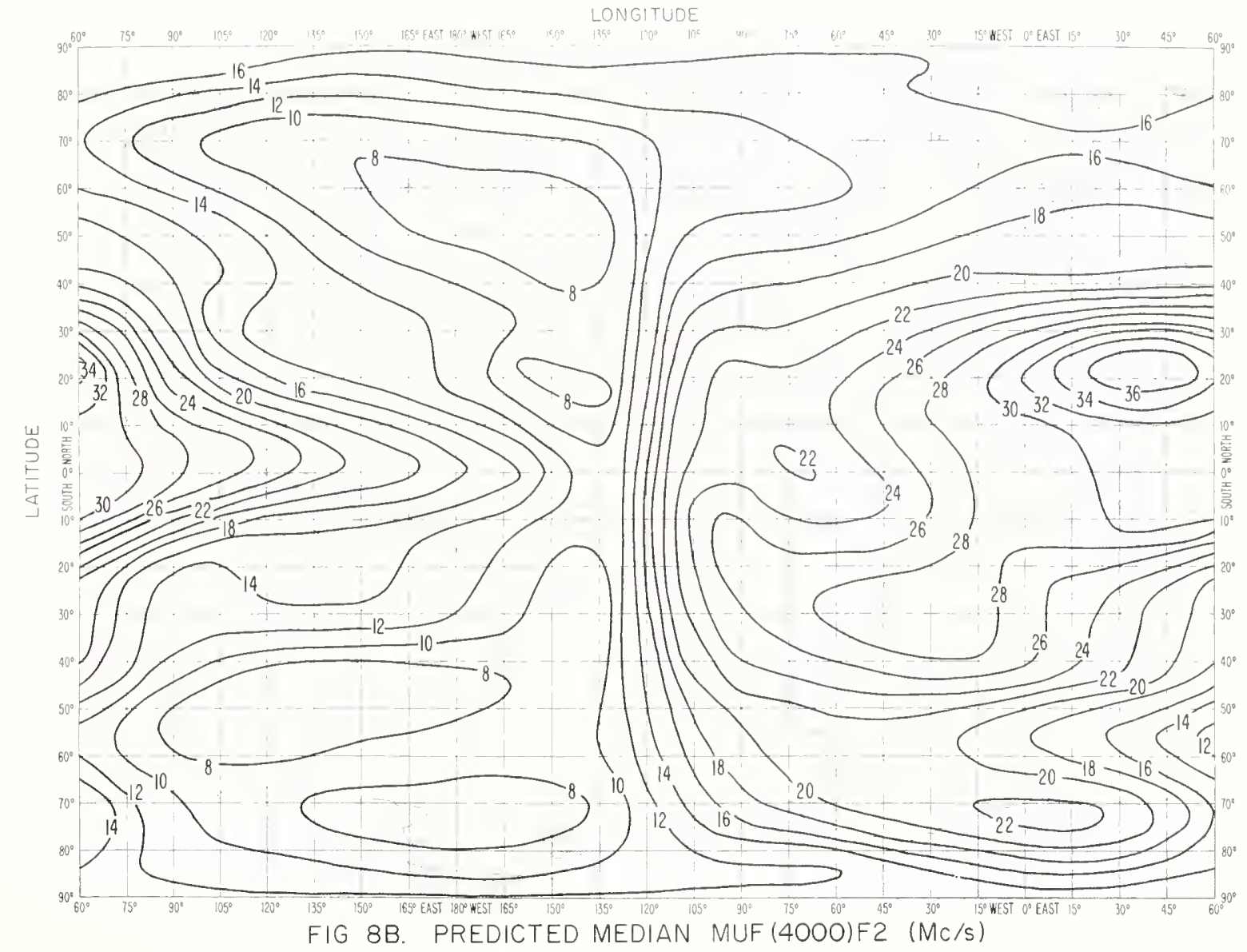




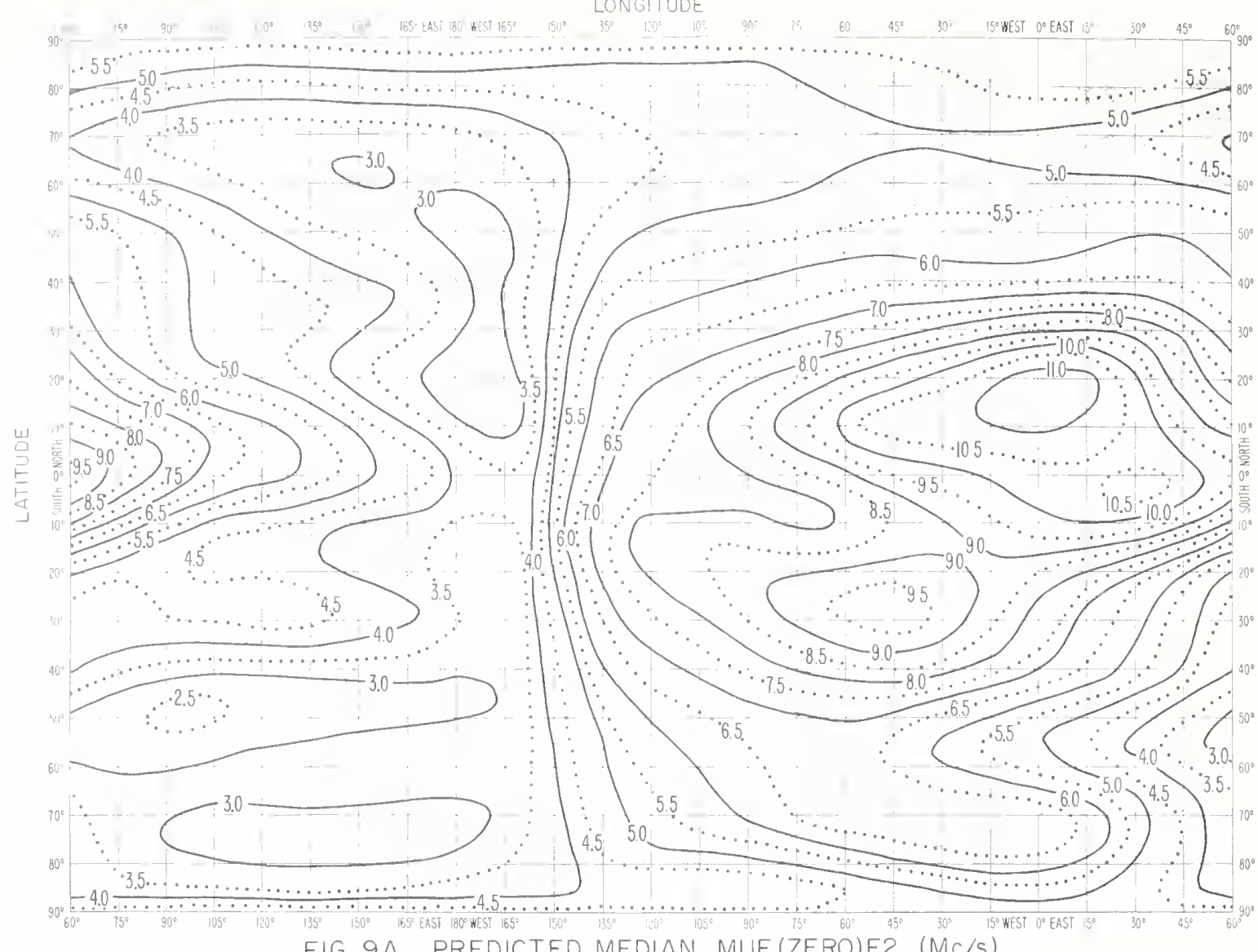

FIG 9A. PREDICTED MEDIAN MUF (ZERO)F2 ( $\mathrm{Mc} / \mathrm{s})$

LONGITUDE

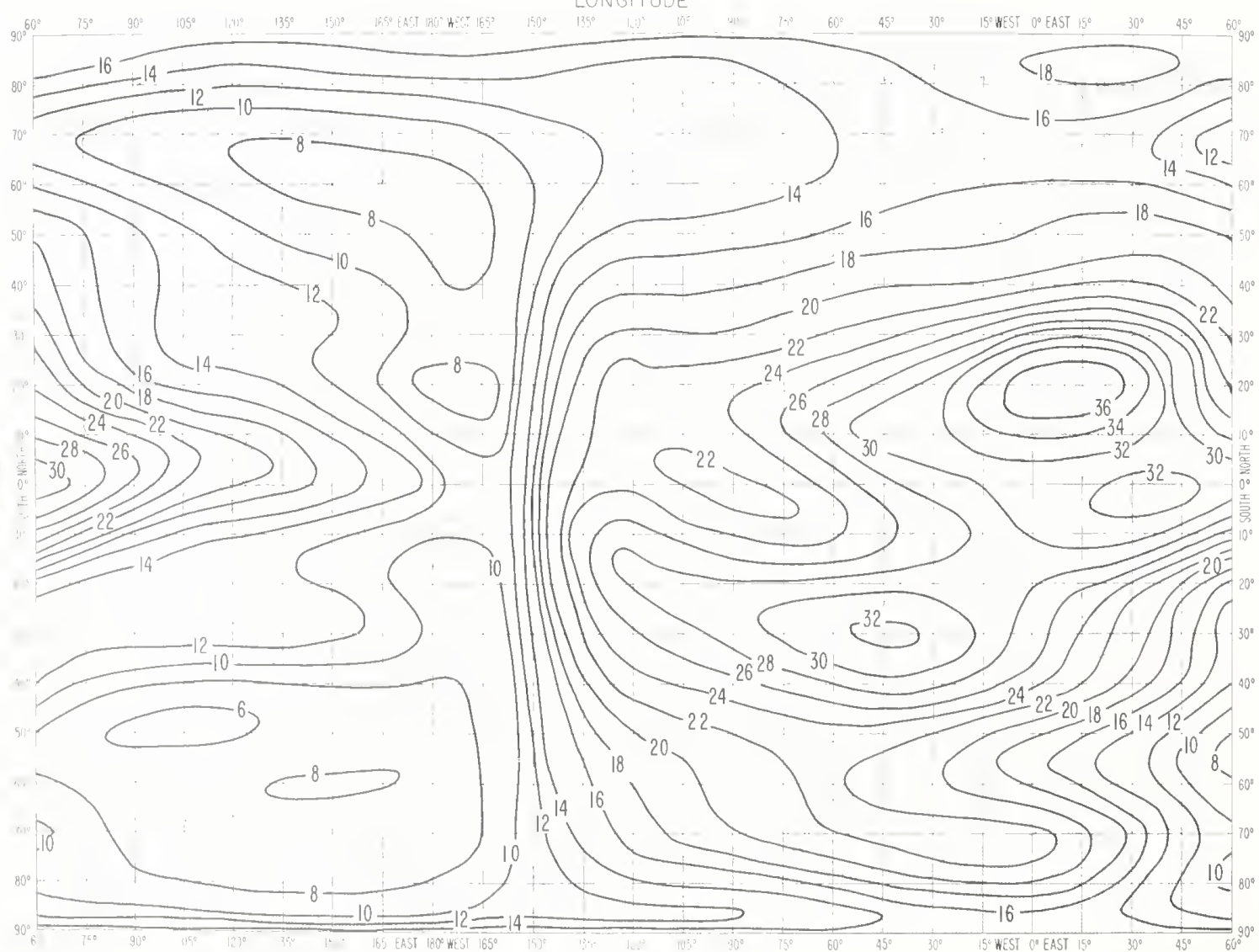

FIG 9B. PREDICTED MEDIAN MUF (4000)F2 ( $\mathrm{Mc} / \mathrm{s})$ 

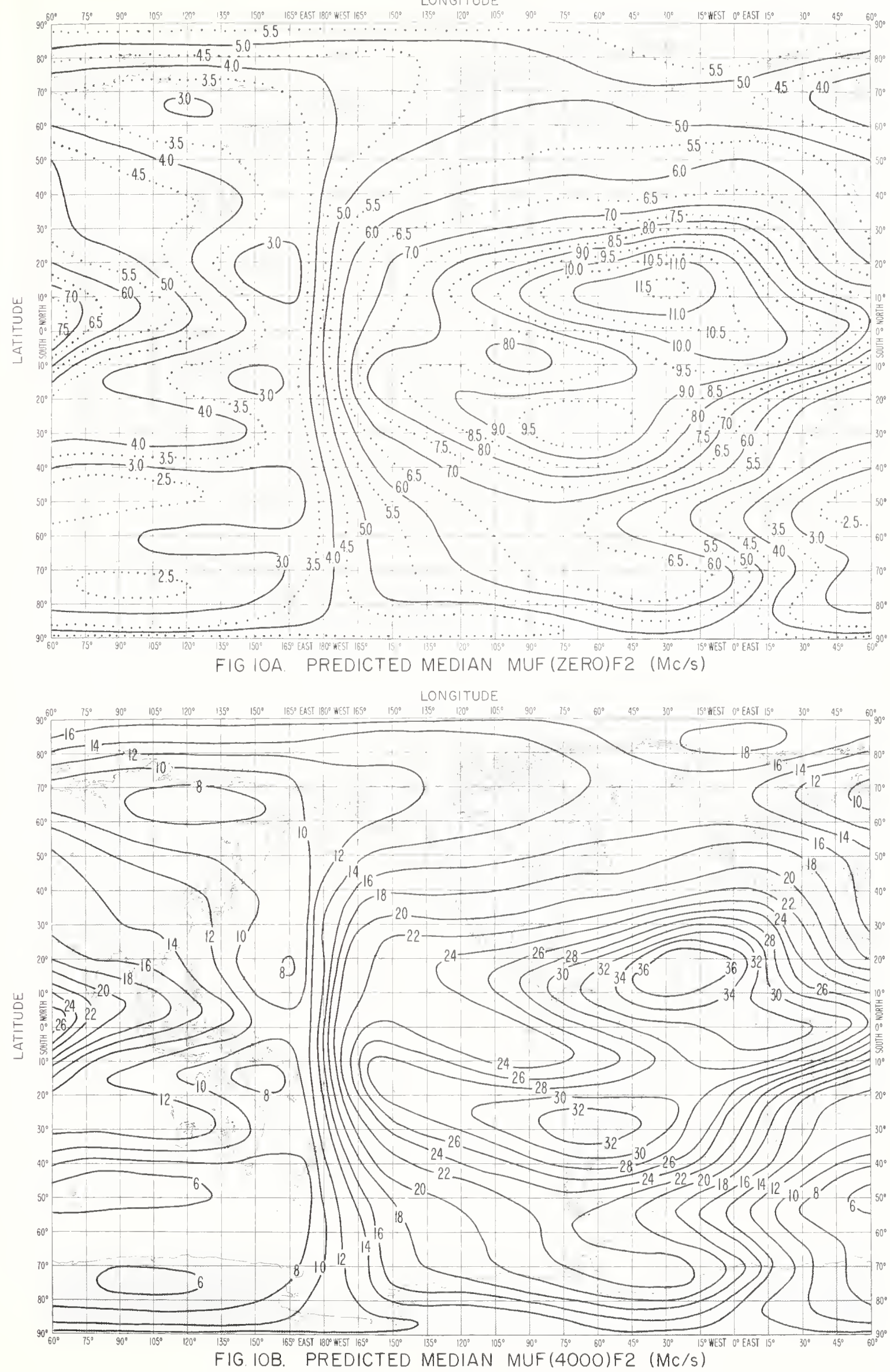


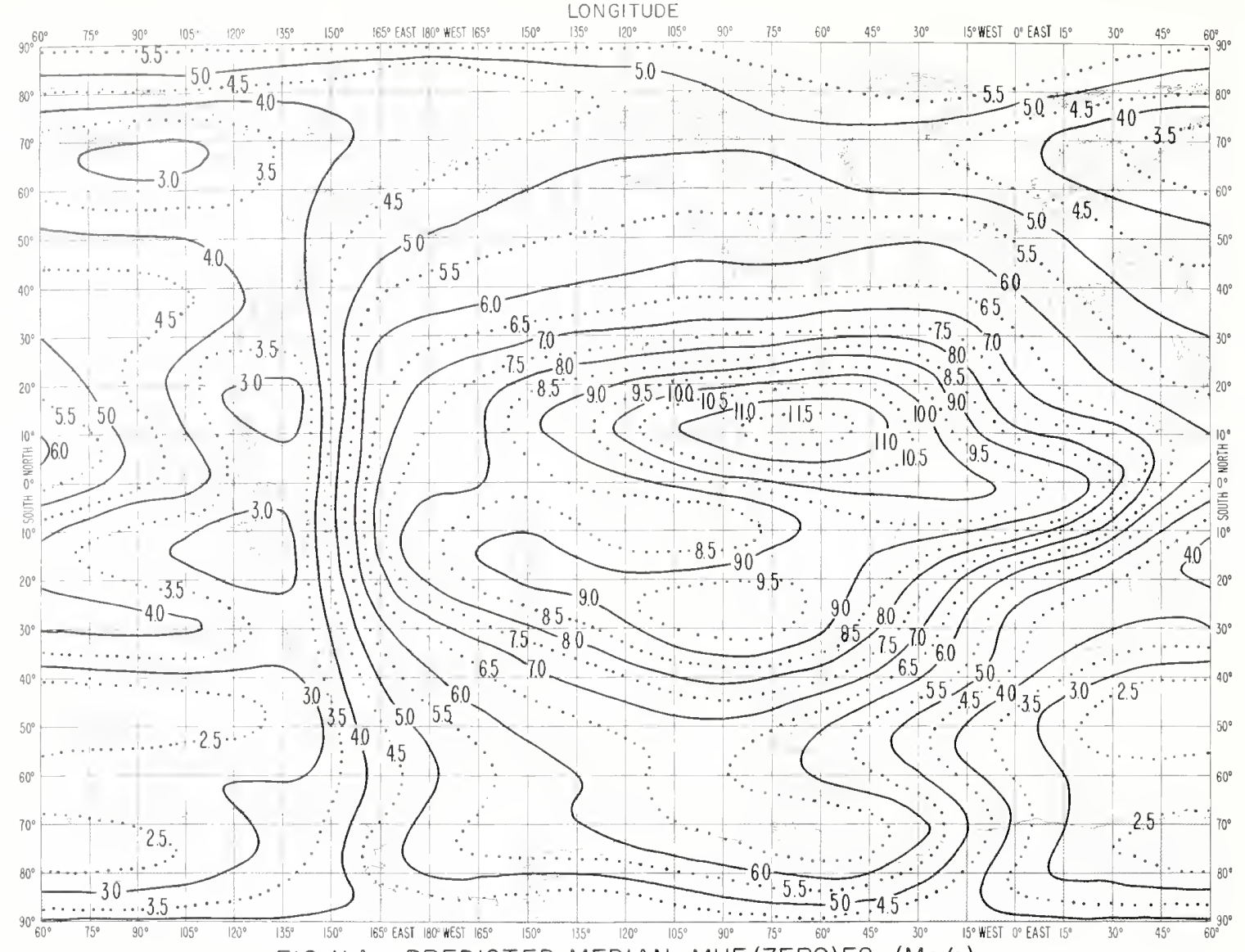

FIG IIA. PREDICTED MEDIAN MUF (ZERO)F2 ( $\mathrm{Mc} / \mathrm{s})$

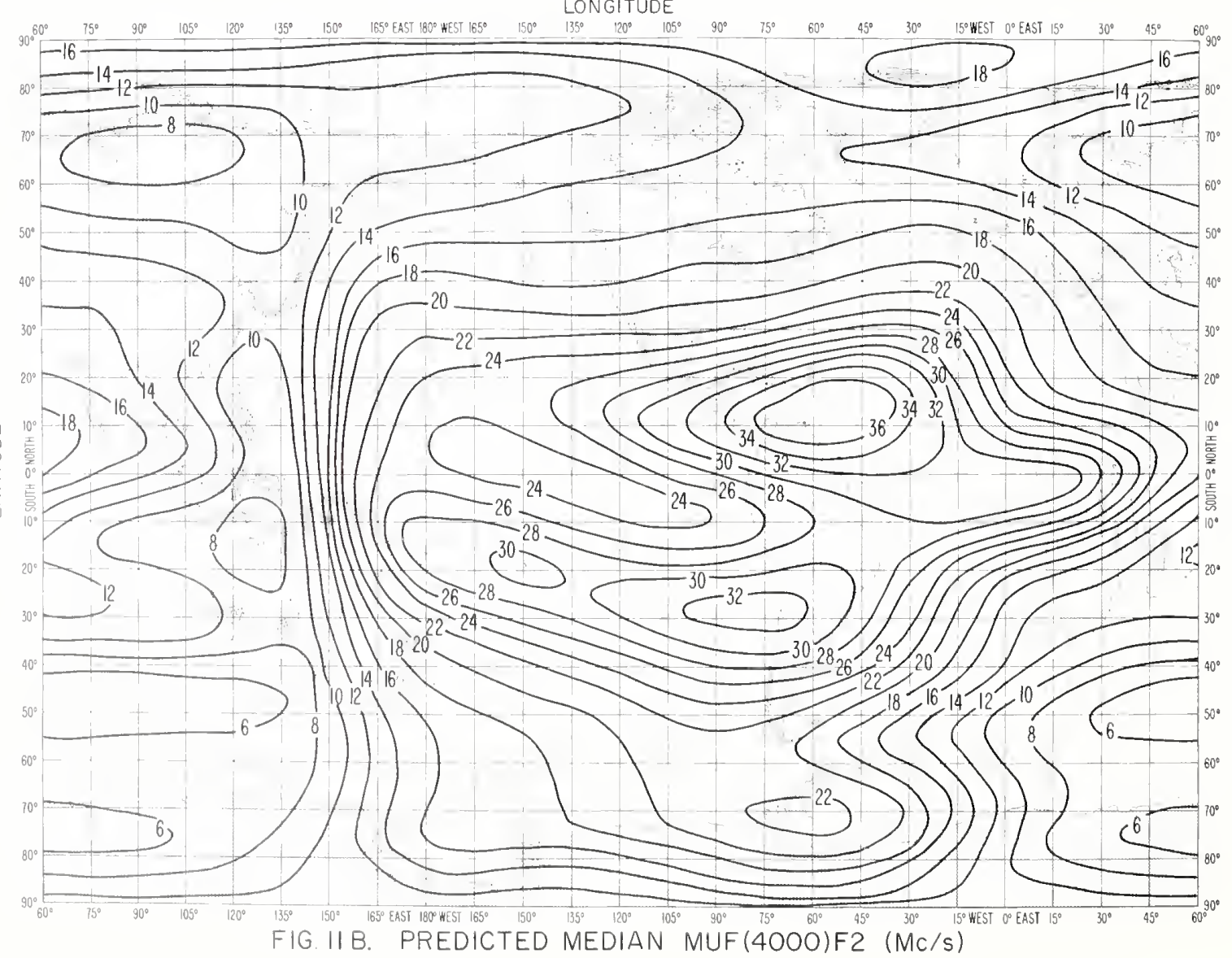




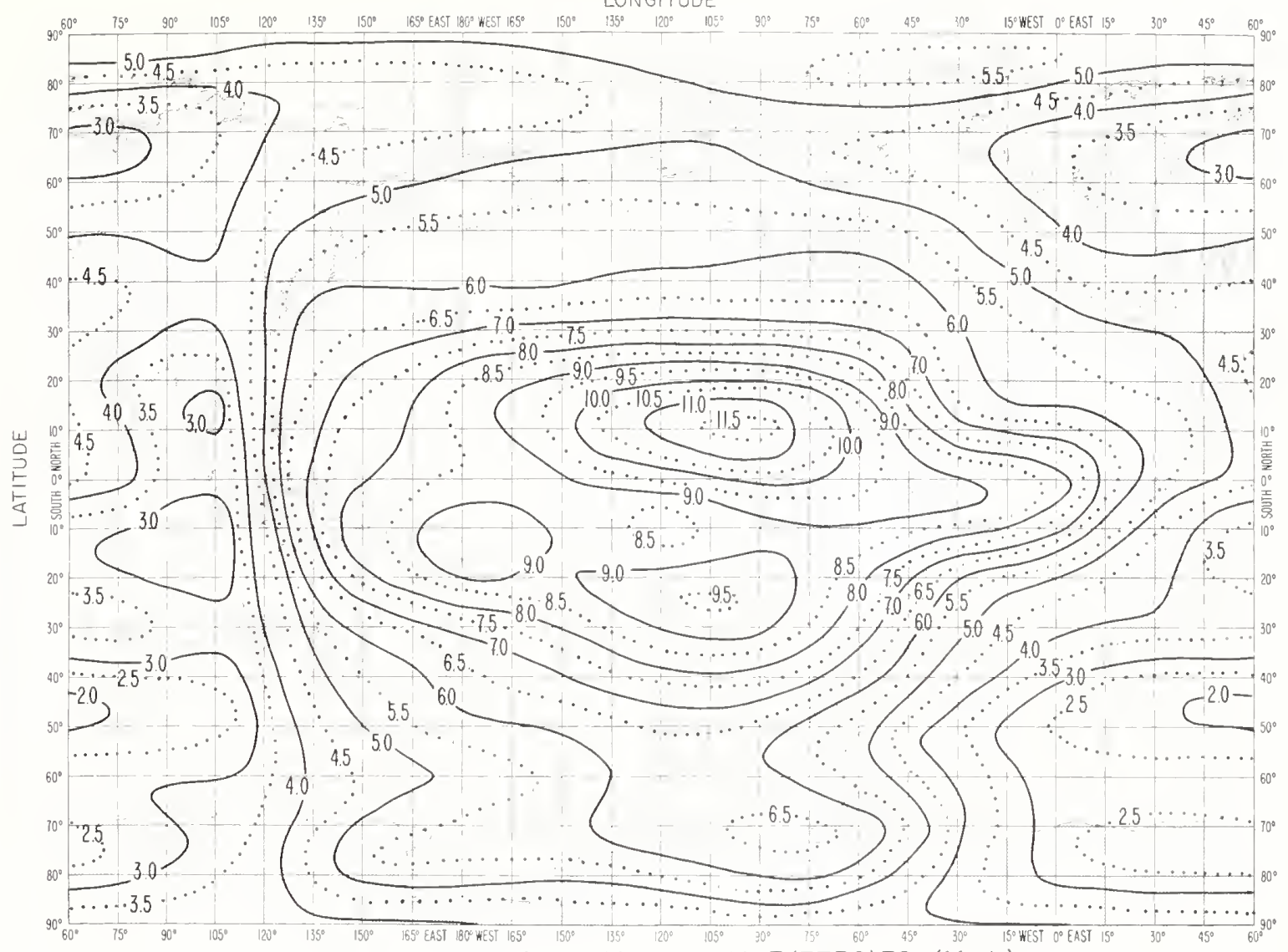

FIG I2A. PREDICTED MEDIAN MUF (ZERO)F2 ( $\mathrm{Mc} / \mathrm{s})$

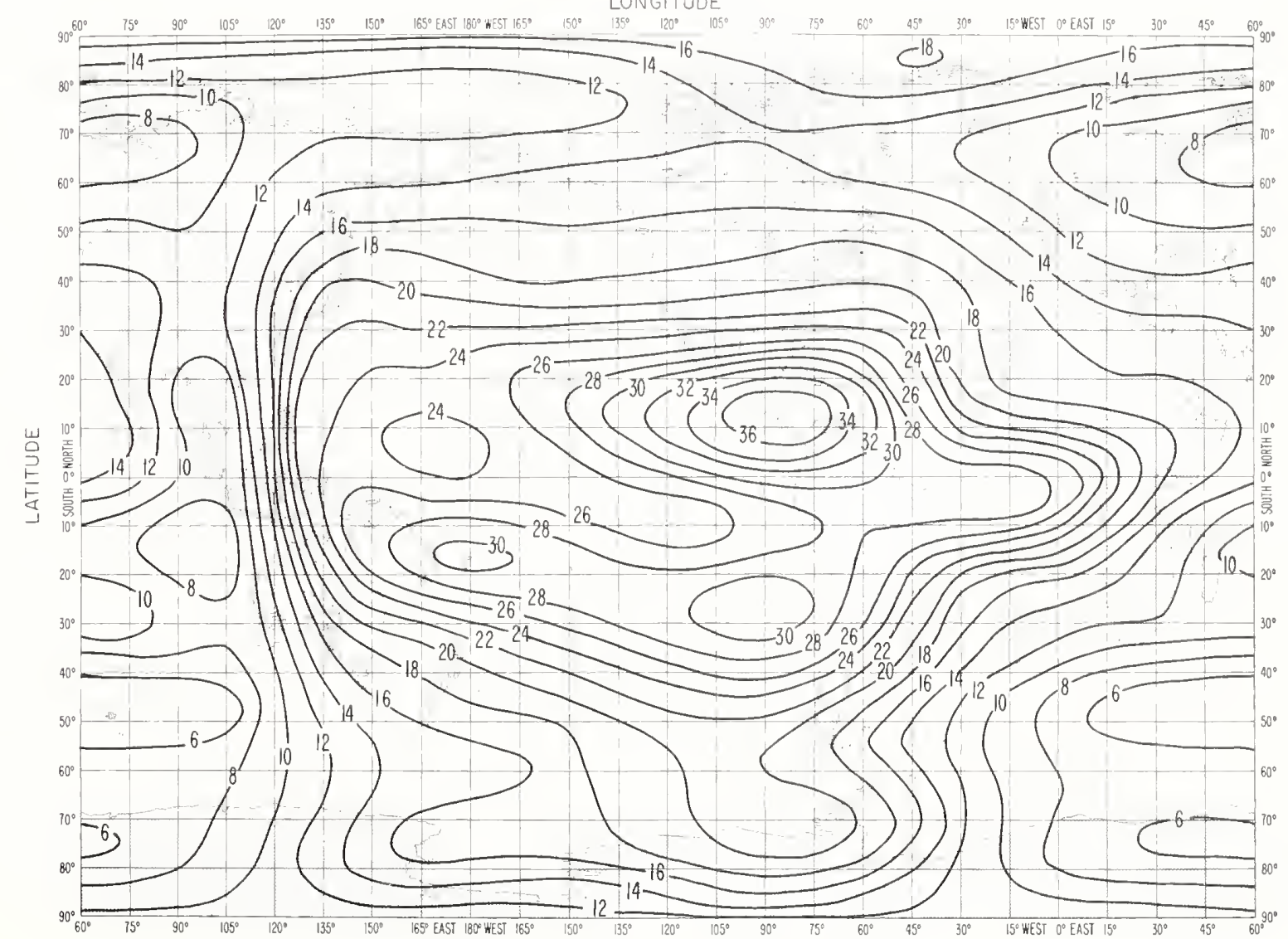

FIG.I2B. PREDICTED MEDIAN MUF(4000)F2 ( $\mathrm{Mc} / \mathrm{s})$ 
NORTH POLAR AREA

SEPTEMBER 1963 UT OO

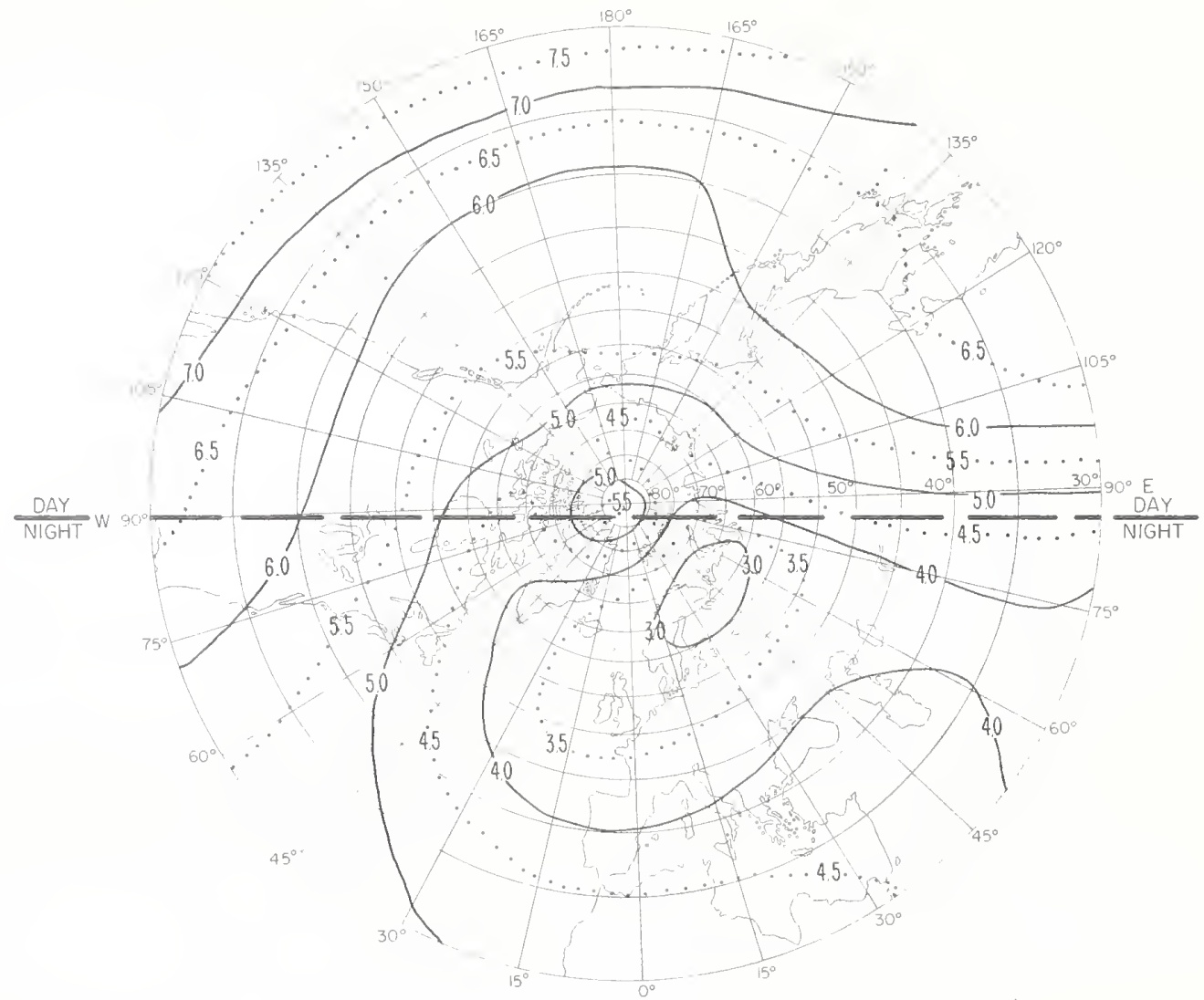

FIG 13A. PREDICTED MEDIAN MUF(ZERO)F2 (MC/S)

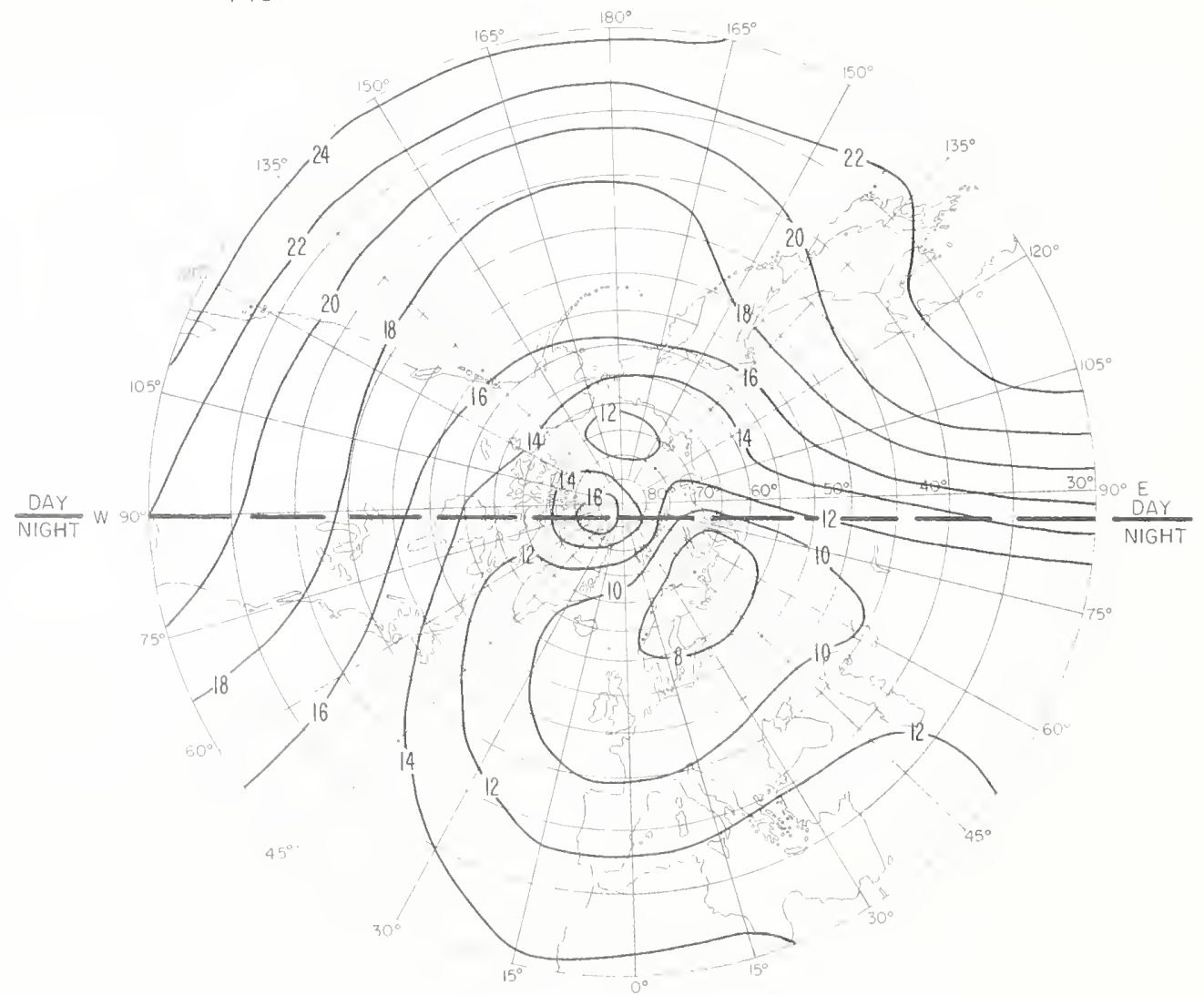

FIG. 13B. PREDICTED MEDIAN MUF (4000)F2 (Mc/s) 


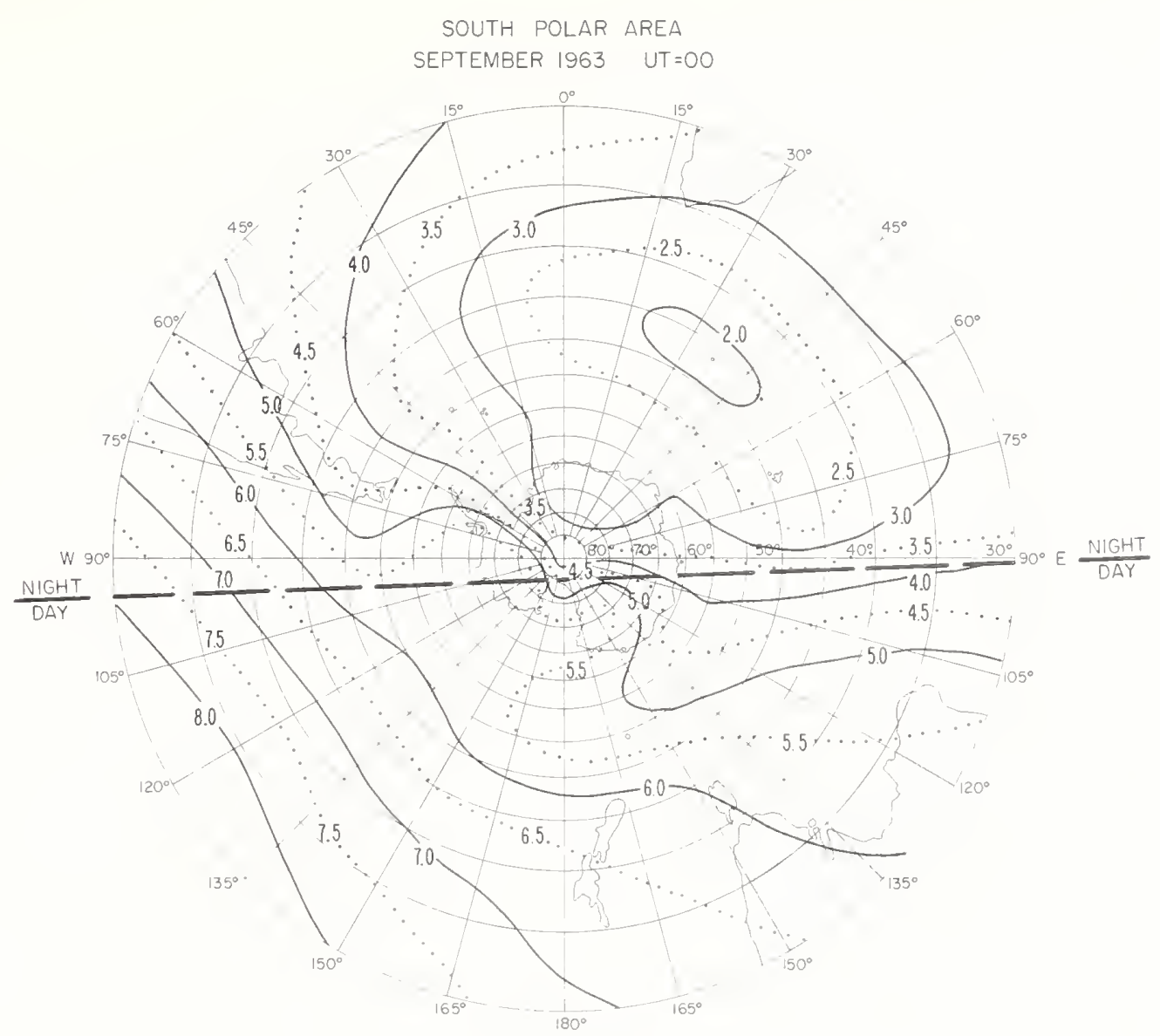

FIG I4A. PREDICTED MEDIAN MUF(ZERO)F2 (Mc/s)

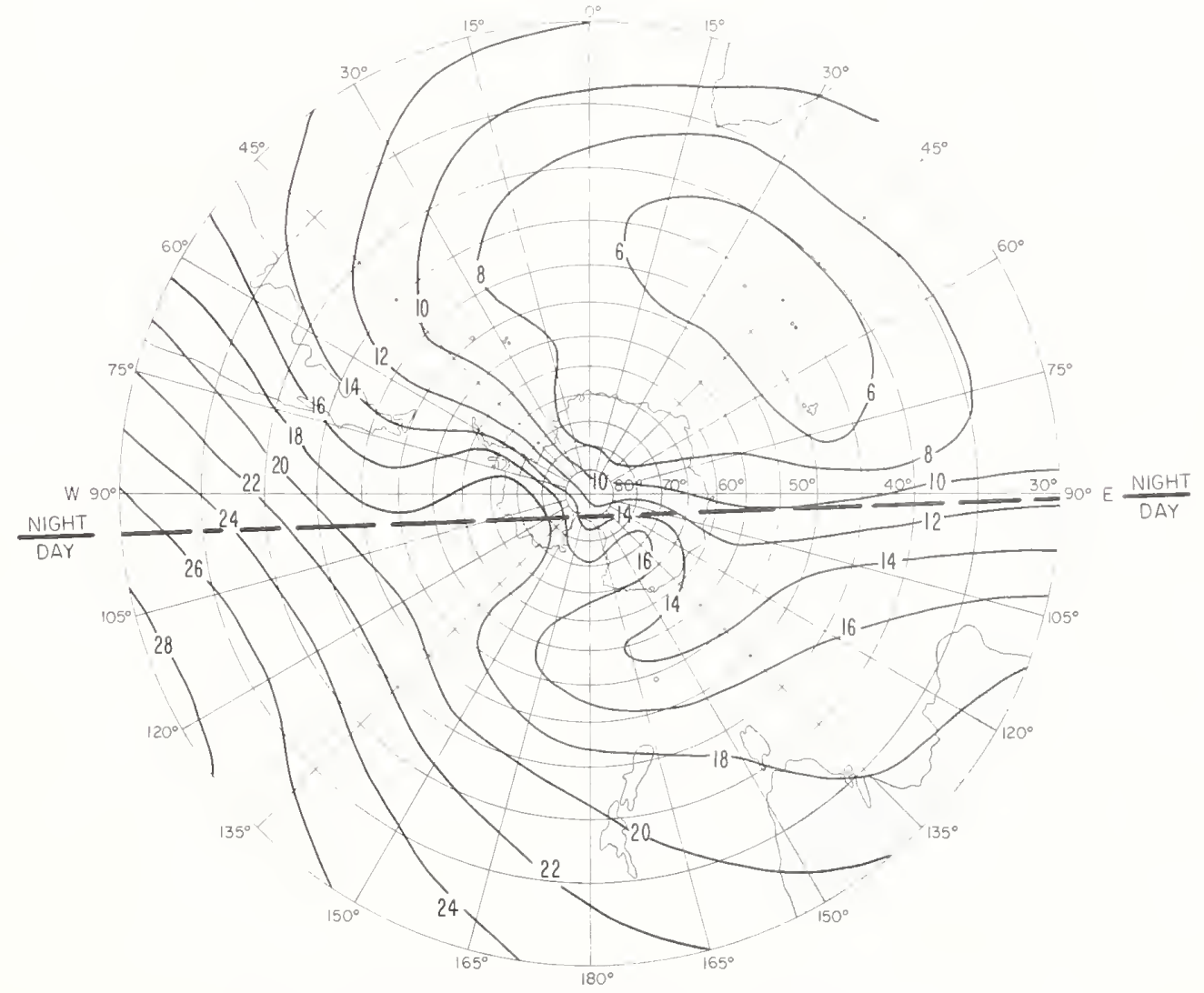

FIG. 14B. PREDICTED MEDIAN MUF(4000)F2 (Mc/s) 


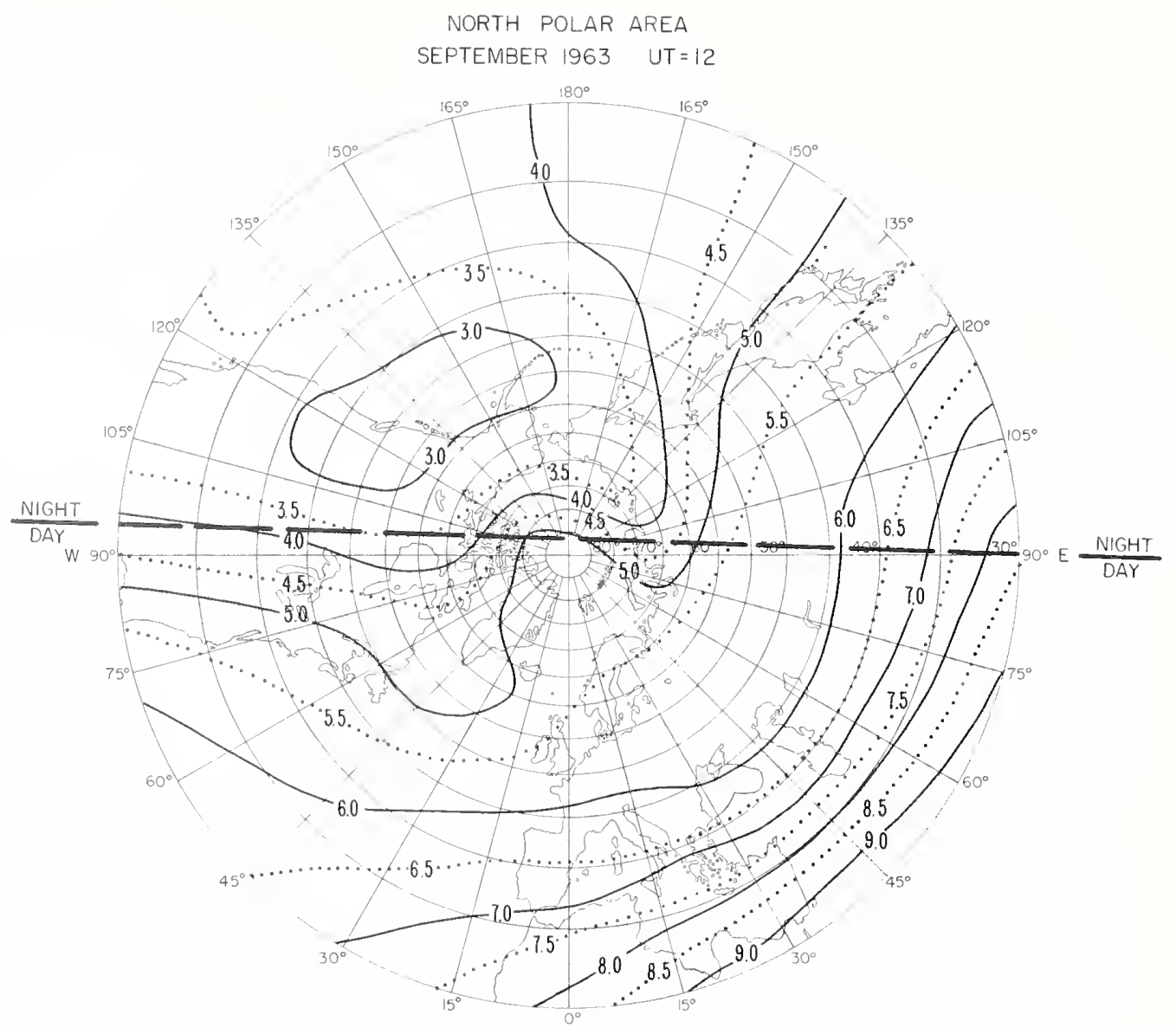

FIG. I5A. PREDICTED MEDIAN MUF (ZERO)F2 (Mc/s)

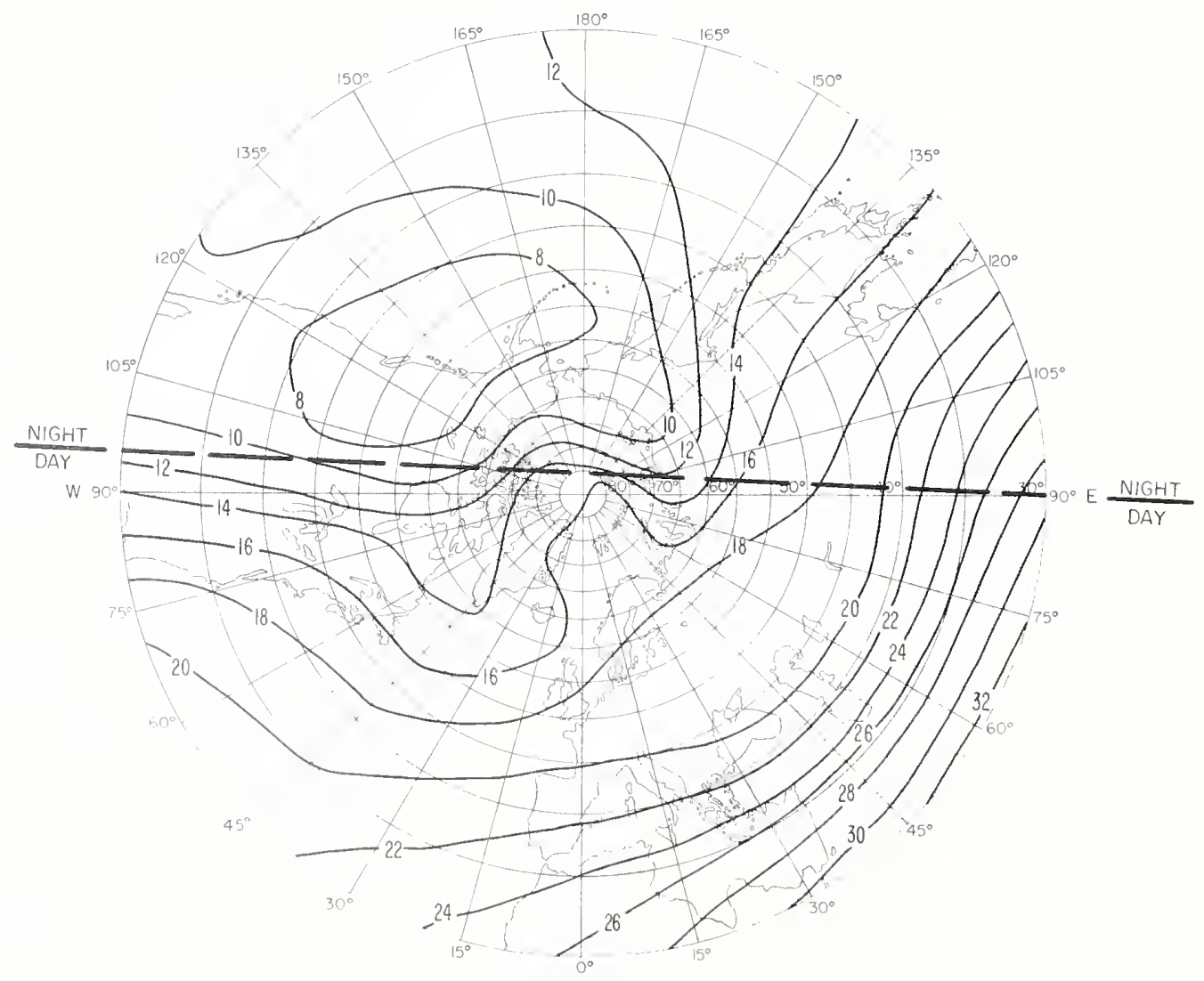

FIG. 15B. PREDICTED MEDIAN MUF (4000)F2 (Mc/s) 


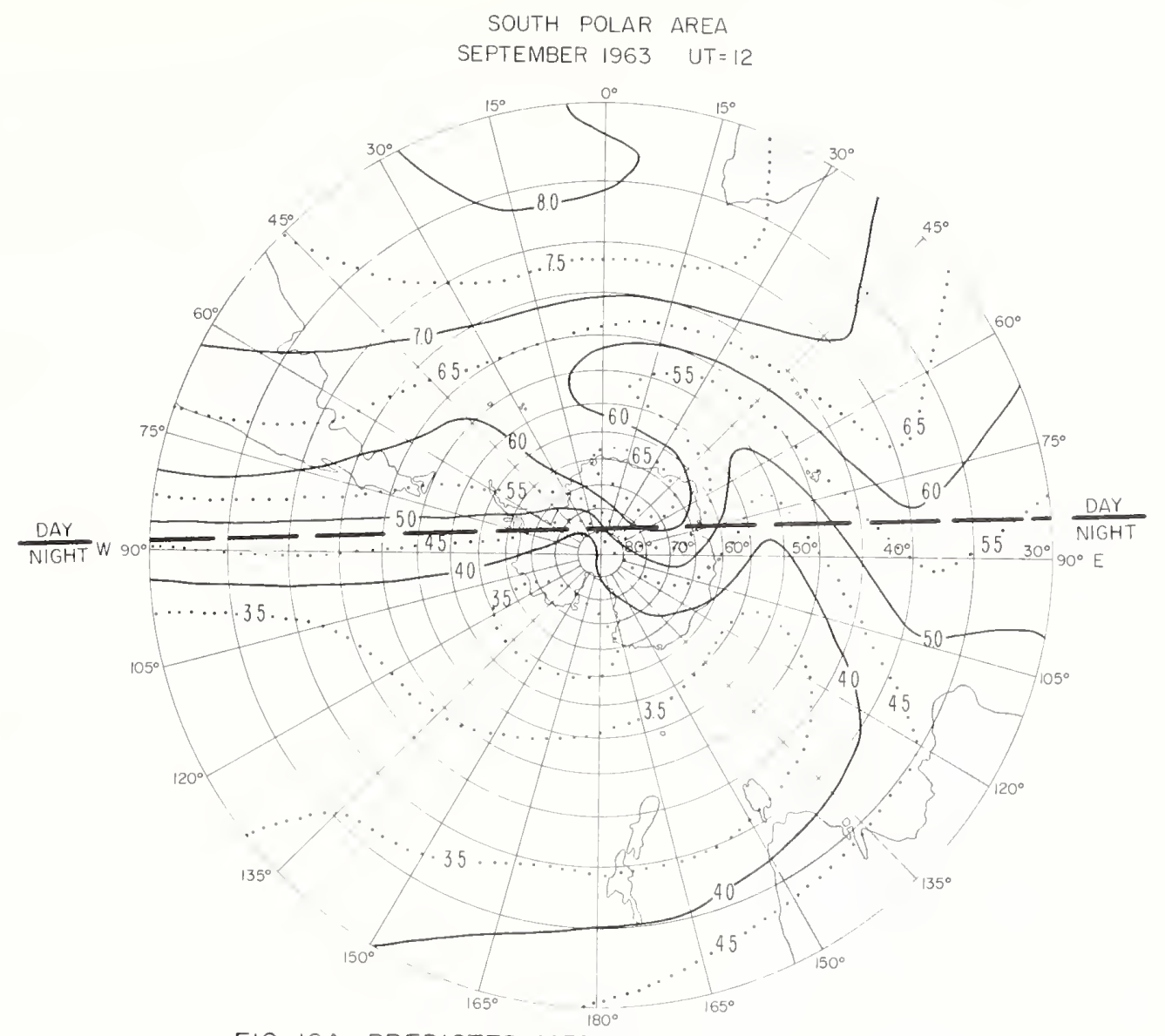

FIG. 16A. PREDICTED MEDIAN MUF(ZERO)F2 (Mc/s)

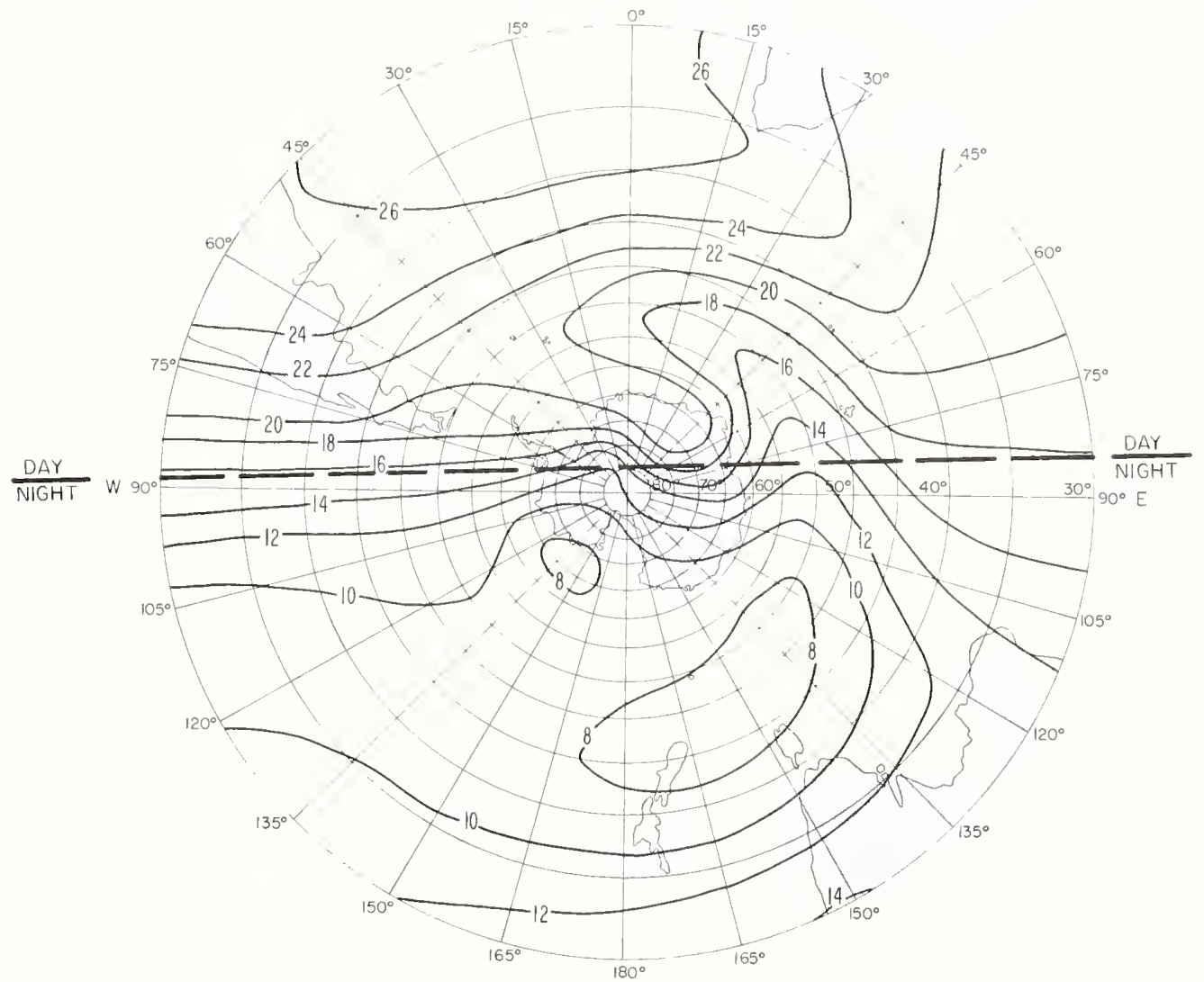

FIG. 16B. PREDICTED MEDIAN MUF(4000)F2 (Mc/s) 



\section{An Indispensable New Instruction Book}

\section{H A N D B O O K \\ for \\ CRPL IONOSPHERIC PREDICTIONS}

Based on Numerical Methods of Mapping

NBS Handbook 90 - by S. M. Ostrow -58 pages - December $1962-\$ 0.40$

All persons engaged in the solution of high frequency radio propagation problems will need this new Handbook, which replaces Instructions for the Use of Basic Radio Propagation Predictions, Circular 465 of the National Bureau of Standards.

Beginning with the January 1963 issue, the monthly radio propagation predictions from the Central Radio Propagation Laboratory of the National Bureau of Standards at Boulder, Colorado, will be entitled CRPL Ionospheric Predictions, instead of Basic Radio Propagation Predictions (CRPL-D Series), as now called.

CRPL Ionospheric Predictions will fulfill the same functions as its predecessor. However, this new version will be prepared by numerical mapping methods, using an electronic computer. The predictions will be presented in two forms, giving the user the choice of either computer or graphical methods. Those using a computer will derive maximum benefits from the system. However, even when a computer cannot be used, the new prediction maps will provide more information than the earlier zone prediction charts, which were prepared by manual methods and designed primarily for graphical solution of high frequency propagation problems.

Thus the new Handbook is a necessity. All users of the CRPL Ionospheric Predictions should discard the now-obsolete Instructions and obtain a copy of the Handbook.

Use the order form below. Please order promptly so that you will have your Handbook by the time the January issue of Predictions arrives.

Superintendent of Documents

U.S. Government Printing Office

Washington 25, D.C.

Enclosed find $\$$ in check $\square$ money order $\square$ for which please send copies of NBS Handbook 90, Handbook for CRPL Ionospheric Predictions to:

Name

Organization

Address

City Zone State (or Country)

(Please Note: Remittances from foreign.countries should be by international money order or by draft on a U.S. bank.) 



\section{THE NATIONAL BUREAU OF STANDARDS}

The scope of activities of the National Bureau of Standards at its major laboratories in Washington, D.C., and Boulder, Colorado, is suggested in the following listing of the divisions and sections engaged in technical work. In general, each section carries out specialized research, development, and engineering in the field indicated by its title.

\section{WASHINGTON, D. C.}

Electricity. Resistance and Reactance. Electrochemistry. Electrical Instruments, Magnetic Measurements. Dielectrics. High Voltage. Absolute Electrical Measurements.

Metrology. Photometry and Colorimetry. Refractometry. Photographic Research. Length. Engineering Metrology. Mass and Scale. Volumetry and Densimetry.

Heat. Temperature Physics. Heat Measurements. Cryogenic Physics. Equation of State. Statistical Physics. Radiation Physics. X-ray. Radioactivity. Radiation Theory. High Energy Radiation. Radiological Equipment. Nucleonic Instrumentation. Neutron Physics.

Analytical and Inorganic Chemistry. Pure Substances. Spectrochemistry. Solution Chemistry. Standard Reference Materials. Applied Analytical Research. Crystal Chemistry.

Mechanics. Sound. Pressure and Vacuum. Fluid Mechanics. Engineering Mechanics. Rheology. Combustion Controls.

Polymers. Macromolecules: Synthesis and Structure. Polymer Chemistry. Polymer Physics. Polymer Characterization. Polymer Evaluation and Testing. Applied Polymer Standards and Research. Dental Research. Metallurgy. Engineering Metallurgy. Microscopy and Diffraction. Metal Reactions. Metal Physics. Electrolysis and Metal Deposition.

Inorganic Solids. Engineering Ceramics. Glass. Solid State Chemistry. Crystal Growth. Physical Properties. Crystallography.

Building Research. Structural Engineering. Fire Research. Mechanical Systems. Organic Building Materials. Codes and Safety Standards. Heat Transfer. Inorganic Building Materials. Metallic Building Materials.

Applied Mathematics. Numerical Analysis. Computation. Statistical Engineering. Mathematical Physics. Operations Research.

Data Processing Systems. Components and Techniques. Computer Technology. Measurements Automation. Engineering Applications. Systems Analysis.

Atomic Physics. Spectroscopy. Infrared Spectroscopy. Far Ultraviolet Physics. Solid State Physics. Electron Physics. Atomic Physics. Plasma Spectroscopy.

Instrumentation. Engineering Electronics. Electron Devices. Electronic Instrumentation. Mechanical Instruments. Basic Instrumentation.

Physical Chemistry. Thermochemistry. Surface Chemistry. Organic Chemistry. Molecular Spectroscopy. Elementary Processes. Mass Spectrometry. Photochemistry and Radiation Chemistry.

Office of Weights and Measures.

\section{BOULDER, COLO.}

Cryogenic Engineering Laboratory. Cryogenic Equipment. Cryogenic Processes. Properties of Materials. Cryogenic Technical Services.

\section{CENTRAL RADIO PROPAGATION LABORATORY}

Ionosphere Research and Propagation. Low Frequency and Very Low Frequency Research. Ionosphere Research. Prediction Services. Sun-Earth Relationships. Field Engineering. Radio Warning Services. Vertical Soundings Research.

Radio Propagation Engineering. Data Reduction Instrumentation. Radio Noise. Tropospheric Measurements. Tropospheric Analysis. Propagation-Terrain Effects. Radio-Meteorology. Lower Atmosphere Physics. Radio Systems. Applied Electromagnetic Theory. High Frequency and Very High Frequency Research. Frequency Utilization. Modulation Research. Antenna Research. Radiodetermination.

Upper Atmosphere and Space Physics. Upper Atmosphere and Plasma Physics. High Latitude Ionosphere Physics. Ionosphere and Exosphere Scatter. Airglow and Aurora. Ionospheric Radio Astronomy.

\section{RADIO STANDARDS LABORATORY}

Radio Physics. Radio Broadcast Service. Radio and Microwave Materials. Atomic Frequency and Time-Interval Standards. Radio Plasma. Millimeter-Wave Research.

Circuit Standards. High Frequency Electrical Standards. High Frequency Calibration Services. High Frequency Impedance Standards. Microwave Calibration Services. Microwave Circuit Standards. Low Frequency Calibration Services. 
UNITED STATES

GOVERNMENT PRINTING OFFICE

DIVISION OF PUBLIC DOCUMENTS

WASHINGTON 25, D. C.

OFFICIAL BUSINESS
PENALTY FOR PRIVATE USE TO AVOID

PAYMENT OF POSTAGE. $\$ 300$ (GPO)

\section{DEPARTMENTS OF THE ARMY \\ AND THE AIR FORCE}

Washington 25, D.C., 1 June 1963

TB 11-499-6/TO 31-3-28, CRPL Ionospheric Predictions for September 1963, published for the information and guidance of all concerned.

By Order of the Secretaries of the Army ANd the Air Force:

EARLE G. WHEELER, General, United States Army, Chicf of Staff.

OFFICIAL:

J. C. LAMBERT,

Major General, United States Army,

The Adjutant General.

OFFICIAL:

$$
\text { R. J. PUGH, }
$$

Colonel, United States Air Forec, Direetor of Administrative Scrviees.

DISTRIBUTION :

Active Army:

USASA (4); USA Elct Comd (1); CSigO (1); USA MI Comd (1); USA Test \& Eval Comd (1); USACD Agcy (1); USA Msl Spt Comd (1); USCONARC (3); ARADCOM (2); OS Maj Comd (5); OS Base Conid (2); Log Cond (2); MDW (1); Amies (5); Corps (2); Div (2); USA Elct RD Agcy (4); Sve Colleges (4); Br Sve Sch (4) except USASCS (20); USAADCEN (2); ARADCOM Rgn (2); WSMR (2); USA Elet RD Actv (4); JBUSMC (12); USA Corps (1); USA Mob Spt Cen (1); USA Sig Msl Spt Comel (1); USAEPG (2); Units org under fol. TOE: 11-18 (1); 11-95 (1); $11-500$ AC (1).

$N G:$ None.

USAR: None.

For explanation of abbreviations used, see A R 320-50. 